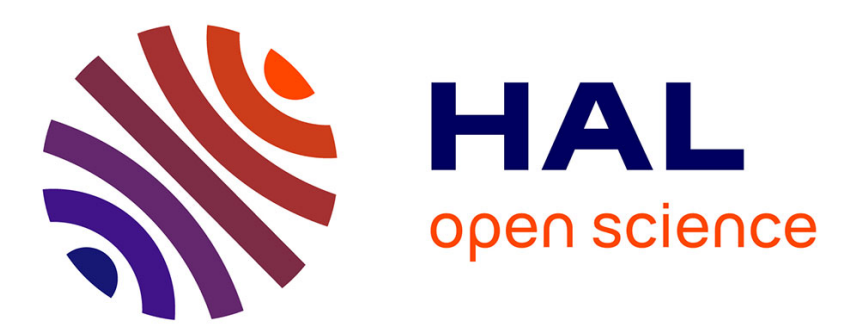

\title{
Cyclic steady states in diffusion-induced plasticity with applications to lithium-ion batteries
}

\author{
Michaël Peigney
}

\section{To cite this version:}

Michaël Peigney. Cyclic steady states in diffusion-induced plasticity with applications to lithium-ion batteries. Journal of the Mechanics and Physics of Solids, 2018, 111, pp.530-556. 10.1016/j.jmps.2017.12.003 . hal-01745344v2

\section{HAL Id: hal-01745344 https://hal-enpc.archives-ouvertes.fr/hal-01745344v2}

Submitted on 9 Apr 2018 (v2), last revised 28 May 2021 (v3)

HAL is a multi-disciplinary open access archive for the deposit and dissemination of scientific research documents, whether they are published or not. The documents may come from teaching and research institutions in France or abroad, or from public or private research centers.
L'archive ouverte pluridisciplinaire HAL, est destinée au dépôt et à la diffusion de documents scientifiques de niveau recherche, publiés ou non, émanant des établissements d'enseignement et de recherche français ou étrangers, des laboratoires publics ou privés. 


\title{
Cyclic steady states in diffusion-induced plasticity with applications to lithium-ion batteries
}

\author{
Michaël Peigney \\ Université Paris-Est, Laboratoire Navier (Ecole des Ponts ParisTech, IFSTTAR, CNRS) , 6-8 avenue \\ Blaise Pascal, Champs-sur-Marne, 77455 Marne la vallée cedex 2, France
}

\begin{abstract}
Electrode materials in lithium-ion batteries offer an example of medium in which stress and plastic flow are generated by the diffusion of guest atoms. In such a medium, deformation and diffusion are strongly coupled processes. For designing electrodes with improved lifetime and electro-mechanical efficiency, it is crucial to understand how plasticity and diffusion evolve over consecutive charging-recharging cycles. With such questions in mind, this paper provides general results for the large-time behavior of media coupling plasticity with diffusion when submitted to cyclic chemo-mechanical loadings. Under suitable assumptions, we show that the stress, the plastic strain rate, the chemical potential and the flux of guest atoms converge to a cyclic steady state which is largely independent of the initial state. A special emphasis is laid on the special case of elastic shakedown, which corresponds to the situation where the plastic strain stops evolving after a sufficiently large number of cycles. Elastic shakedown is expected to be beneficial for the fatigue behavior and - in the case of lithium-ion batteries - for the electro-chemical efficiency. We provide a characterization of the chemo-mechanical loadings for which elastic shakedown occurs. Building on that characterization, we suggest a general method for designing structures in such fashion that they operate in the elastic shakedown regime, whatever the initial state is. An attractive feature of the proposed method is that incremental analysis of the fully coupled plasticity-diffusion problem is avoided. The results obtained are applied to the model problem of a battery electrode cylinder particle under cyclic charging. Closed-form expressions are obtained for the set of charging rates and charging amplitudes for which elastic shakedown occurs, as well as for the corresponding cyclic steady states of stress, lithium concentration and chemical potential. Some results for a spherical particle are also presented.
\end{abstract}

Keywords: lithium-ion batteries, diffusion-induced stress, shakedown, plasticity, chemo-mechanical coupling, cyclic steady state

Email address: michael.peigney@polytechnique.org (Michaël Peigney)

Preprint submitted to Journal of $E^{A} T_{E} X$ Templates

April 9, 2018 


\section{Introduction}

In In lithium-ion batteries, the flow of electrons results from the transport of lithium ions in an electrolyte and their diffusion in solid electrodes. The intercalation of lithium in the electrode material results in a local volumetric change which may in turn produce some stress, in a way similar to thermal stress. Diffusion-induced stress may lead to fracture and thus contribute to the power loss and the capacity fading of batteries (Huggins and Nix, 2000).

The critical role of diffusion-induced stress in lithium-ion batteries has motivated a lot of work in the modeling community. Most models assume a linear elastic behavior for the electrode material, so that diffusion is the only dissipative process. In some models, the diffusion problem is further decoupled from the mechanical problem by assuming that the chemical potential - which is the thermodynamical force driving diffusion - is independent of the stress (Zhao et al., 2010). Under such an assumption, analytical expressions can be obtained for electrode particles with simple geometries (Cheng and Verbrugge, 2009). More realistic models account for full coupling between diffusion and mechanics by appealing to the consistent thermodynamical framework introduced by Larché and Cahn (1973). Numerical results have been obtained for various geometries (Christensen and Newman, 2006; Zhang et al., 2007; Swaminathan et al., 2016) but no analytical expression has been obtained so far.

For spherical or cylindrical electrode particles, the mentioned elastic models show that some stress concentration occurs at fast charging. In such a condition, the yield limit of the electrode material may be reached so that plasticity needs to be taken into account. Models coupling plasticity with diffusion have notably been considered by Bower et al. (2011); Zhao et al. (2011); Cui et al. (2012); Brassart et al. (2013) and Yang et al. (2014). From a thermodynamic point of view, plastic flow and diffusion act as two concurrent dissipative processes. The interplay between plasticity and diffusion may lead to some unusual behavior: For instance, under a monotone loading, a particle may start to flow plastically and then unload elastically after some time (Brassart et al., 2013).

Since improving life cycle and durability is a major concern in the design of lithium-ion batteries, it is essential to understand how plasticity and diffusion evolve over many cycles of lithiation-delithiation. Using numerical simulations, Brassart et al. (2013) studied the behavior of a spherical elastic-plastic particle under cyclic charging. For a charging at high rate or high amplitude, a steady state regime of cyclic plasticity is observed. For a charging at low rate and low amplitude, the plastic strain stops evolving after a few cycles so that the particle evolves elastically in subsequent cycles. This last behavior is known as elastic shakedown in plasticity theory. The results of Brassart et al. (2013) were obtained for a special geometry and using an initial natural state. For other geometries such as thin films, a ratcheting behavior can be observed at fast charging, i.e. there is an unbounded accumulation of plastic strain over consecutive lithiation-delithiation cycles, leading to early failure (Haftbaradaran and Gao, 2012). Those types of plastic behaviors 
are not specific to diffusion-related problems and are also encountered in classical elasticplastic structures. In that last case, it is known that the stress and the plastic strain rate alway: 1 converge to a cyclic response as time tends to infinity (Frederick and Armstrong, 1966; Halphen, 1978, Johnson et al., 1978). It is not clear whether a similar result holds for problems coupling plasticity with diffusion. The role of residual stress also needs to be addressed: in real specimen, some residual stress may exist as a result of inelastic deformation due to material process or trapped lithium ions. Such a residual stress has an influence on the plastic flow and the diffusion of lithium over the loading cycles. In particular, the impact of the residual stress on the large-time behavior needs to be clarified.

This paper aims at answering such questions by providing general results regarding the large-time behavior of cyclically loaded media coupling plasticity with diffusion. Although much emphasis is laid on lithium-ion batteries, the results may apply to other problems such as hydrogen embrittlement in metals. The general framework considered is formulated in the geometrically linear setting and is described in Sect.2. Full coupling between diffusion and mechanics is accounted for, in the spirit of Larché and Cahn (1973). Both the plasticity flow rule and the law of diffusion are allowed to be nonlinear. In Sect. 3 we establish the equation (3.8) that governs the evolution of the stress and chemical potential fields. The evolution equation (3.8) is central in our analysis. We first establish some of its properties by extending some results established by Halphen (1978) in standard plasticity. Those properties are used in Sect. 4 for proving some of the main results of this paper: The stress, the chemical potential, the local concentration of guest atoms as well as the plastic strain rate alway: $2^{2}$ converge to a cyclic steady state as time tends to infinity. Moreover, the values reached by the plastic strain rate and the chemical potential in the steady state cycle are independent of the initial state. Those results (see Properties 3 and 4 for the exact statements) have a number of consequences. First, there is no other plastic behavior than the three ones listed previously (shakedown, cyclic plasticity, ratcheting), whatever the structure or the loading. Second, for a given structure, the observed plastic behavior depends only on the loading, i.e. there is no influence of the residual stress. Third, diffusion reaches a cyclic steady state in which the flux and the local concentration of injected atoms are independent of the initial state. For lithium-ion batteries, the charge-voltage response thus converges to a uniquely defined cyclic steady state.

In Sect. 5 we focus on the particular case of elastic shakedown which is arguably the most beneficial with respect to fatigue (see e.g. Constantinescu et al. (2003) and references therein). Our objective is to obtain a priori restrictions on the loading for ensuring that elastic shakedown occurs, in the spirit of Melan theorem in standard plasticity (Melan, 1936 Koiter, 1960; Symonds, 1951). That theorem was originally proposed for elasticperfectly plastic materials and has been extended to several more complex behaviors -

\footnotetext{
${ }^{1}$ under suitable technical assumptions

${ }^{2}$ under some assumptions formulated in Sect. 4, see Property 4
} 
see e.g. Ahn et al. (2008); Peigney (2010, 2014a b); Pham (2008, 2017); Klarbring et al. (2017) and Spagnoli et al. (2017) for some recent examples. The proof used in the original Melan theorem - as well as in most of its extensions - consists in showing that the total energy dissipation remains bounded as time tends to infinity. When plastic flow is the only dissipative process, bounding the total dissipation indeed amounts (at least formally) to requiring that the plastic strain tends to a time-independent limit (see e.g. Nguyen (2003) for more detail). The situation changes when diffusion is introduced: Bounding the total energy dissipation means that the plastic strain tends to a limit and that diffusion reaches an equilibrium (i.e. the local concentration of guest atoms converges to a timeindependent limit). Such a situation is of limited interest for systems such as lithium-ion batteries: One is more interested in bounding the plastic dissipation only, allowing for the total dissipation to remain unbounded as a result of continuous diffusion over consecutive lithiation-delithiation cycles. The arguments at play in Melan theorem do not allow only one part of the total dissipation to be bounded, hence a different line of reasoning is needed. Building on the results of Sect. 4, we establish necessary and sufficient conditions for elastic shakedown to occur - without enforcing that diffusion reaches an equilibrium. Those results could notably be used for the elastic shakedown design of lithium-ion batteries under operative charging conditions, ensuring that the electrode behaves elastically after a run-in period of plastic flow.

The results obtained are illustrated in Sect. 6 on the model problem of a cylinder electrode particle under cyclic lithitation-delithiation. The two loading parameters are the lithiation rate and the total number of injected lithium ions. Explicit expressions are first obtained for the purely elasto-diffusive response of the particle, thus complementing available results in the literature. That elasto-diffusive solution is needed for applying the shakedown conditions, leading to closed-form expressions of the shakedown domain in the space of loading parameters. Some results for a spherical particle are also presented.

\section{Chemo-mechanical evolutions of an elastic-plastic continuum}

\subsection{Conservation equations}

Consider a deformable continuum in which guest atoms diffuse. The local concentration of guest atoms and the displacement (with respect to a reference configuration) are denoted by $c$ and $\boldsymbol{u}$, respectively. The mass conservation leads to the diffusion equation

$$
\dot{c}+\operatorname{div} \boldsymbol{\jmath}=0 \text { in } \Omega,
$$

where $\boldsymbol{J}$ is the flux of guest atoms and $\Omega$ is the domain occupied by the continuum. The superscript ' denotes time differentiation. Eq. 2.1) is complemented by the boundary conditions

$$
\mu=M \text { on } \Gamma_{\mu}, \quad \boldsymbol{J} \cdot \boldsymbol{n}=J \text { on } \Gamma_{J}=\partial \Omega-\Gamma_{\mu}
$$


where $\mu$ is the chemical potential and $\boldsymbol{n}$ is the outward-pointing normal vector. Eq. 2.2 expresses the fact that the chemical potential is required to take a prescribed value $M$ on a part $\Gamma_{\mu}$ of the boundary. On $\Gamma_{J}$, the normal flux $J$ of guest atoms is prescribed.

We restrict our attention to the geometrically linear setting, defining the strain $\varepsilon$ as $\varepsilon=\left(\nabla \boldsymbol{u}+\nabla^{T} \boldsymbol{u}\right) / 2$. Deformation is considered to be much faster than diffusion so that mechanical equilibrium holds at all time. The stress field $\boldsymbol{\sigma}$ thus satisfies the equilibrium equation

$$
\operatorname{div} \boldsymbol{\sigma}+\boldsymbol{f}=0 \text { in } \Omega
$$

where $\boldsymbol{f}$ is the body force. Eq. 2.3 is complemented by the boundary conditions

$$
\boldsymbol{\sigma} \cdot \boldsymbol{n}=\boldsymbol{T} \text { on } \Gamma_{T}, \quad \boldsymbol{u}=\boldsymbol{U} \text { on } \Gamma_{u}=\partial \Omega-\Gamma_{T}
$$

where $\boldsymbol{T}$ are tractions prescribed on a part $\Gamma_{T}$ of the boundary and $\boldsymbol{U}$ are prescribed displacements.

All the functions $M, J, \boldsymbol{f}, \boldsymbol{U}$ and $\boldsymbol{T}$ depend on the location $\boldsymbol{x}$ and time $t$. Similarly, the fields $c, \mu, \boldsymbol{u}$ and $\boldsymbol{\sigma}$ are all functions of $\boldsymbol{x}$ and $t$. Unless in the case of possible ambiguities, the dependence in $(\boldsymbol{x}, t)$ will be omitted in the notations.

\subsection{Constitutive equations}

An elastic-plastic constitutive material is considered. Assuming small perturbations with respect to a reference configuration, the free energy $w$ of the material is taken as a quadratic function of the form

$$
w\left(\varepsilon, \varepsilon^{p}, c\right)=\frac{1}{2}\left(\varepsilon-\varepsilon^{p}\right): \mathbb{L}:\left(\varepsilon-\varepsilon^{p}\right)+\frac{1}{2} k c^{2}+c \boldsymbol{A}:\left(\varepsilon-\varepsilon^{p}\right)+c \mu_{0}
$$

where $\varepsilon^{p}$ is the (deviatoric) plastic strain. In $(2.5), \mathbb{L}$ is the symmetric positive definite fourth-order elasticity tensor and $\boldsymbol{A}$ is a symmetric second-order tensor coupling the elastic strain $\varepsilon-\varepsilon^{p}$ with the concentration $c$. The notation : denotes contraction with respect to the last two indices, e.g. $(\mathbb{L}: \varepsilon)_{i j}=\sum_{k l} \mathbb{L}_{i j k l} \varepsilon_{l k}$. The constant $\mu_{0}$ in 2.5) is a reference value of the chemical potential. The function $w$ in 2.5 can be verified to be convex if the material parameter $k$ satisfies

$$
k-\boldsymbol{A}: \mathbb{L}^{-1}: \boldsymbol{A}>0 .
$$

The condition (2.6) is assumed to be satisfied in the following.

From (2.5) we obtain the expressions of the stress $\boldsymbol{\sigma}$ and the chemical potential $\mu$ as

$$
\begin{aligned}
\boldsymbol{\sigma} & =\frac{\partial w}{\partial \varepsilon}=\mathbb{L}:\left(\varepsilon-\varepsilon^{p}\right)+c \boldsymbol{A}, \\
\mu & =\frac{\partial w}{\partial c}=\mu_{0}+k c+\boldsymbol{A}:\left(\varepsilon-\varepsilon^{p}\right) .
\end{aligned}
$$


It is clear from (2.7) that the coupling between the mechanical variables $\left(\boldsymbol{\sigma}, \boldsymbol{\varepsilon}, \boldsymbol{\varepsilon}^{p}\right)$ and the chemical variables $(c, \mu)$ operates in a two-way fashion. On one hand, the concentration $c$ indeed has a linear influence on the stress, in a way similar to thermal stress. On the other hand, the chemical potential $\mu$ depends linearly on the elastic strain $\varepsilon-\varepsilon^{p}$, which implies that the mechanical state has an influence on the diffusion process, see Eqs. (2.1) and (2.8).

The constitutive equations (2.7) are complemented by a law of diffusion and a plasticity flow rule. In more detail, the flux $\boldsymbol{\jmath}$ of guest atoms is assumed to derive from a differentiable, strictly convex potential $\psi$ through the relation

$$
\boldsymbol{\jmath}=-\partial \psi(\nabla \mu)
$$

Fick's law corresponds to the particular case where $\psi$ is quadratic in $\nabla \mu$. The plasticity flow rule is taken in the classical form

$$
\dot{\varepsilon}^{p} \in \partial \phi(\boldsymbol{\sigma})
$$

where $\phi$ is the dissipation potential. In $(2.8)$ and $(2.9), \partial$ denotes the subdifferential operator (Van Tiel, 1984). Following standard requirements, $\phi$ is assumed to be convex, positive and null at the origin. Under those assumptions, we note for later reference that the flow rule $(2.9)$ implies that

$$
\left(\boldsymbol{\sigma}^{\prime}-\boldsymbol{\sigma}\right): \dot{\boldsymbol{\varepsilon}}^{p} \leq 0 \text { for any } \boldsymbol{\sigma}^{\prime} \in \mathcal{C},
$$

where $\mathcal{C}=\{\boldsymbol{\sigma}: \phi(\boldsymbol{\sigma})=0\}$ is the elasticity domain of the material. The relation 2.10 is known as the principle of maximum dissipation (Hill, 1950).

In this paper we will consider two of the most usual situations in viscoplasticity and rate-independent plasticity :

Viscoplasticity. $\phi$ is a differentiable, convex function of the deviatoric stress and $\phi$ is strictly convex (with respect to the deviatoric stress) outside of the elasticity domain $\mathcal{C}$.

Rate-independent plasticity. $\phi$ is infinite outside of $\mathcal{C}$ and the elasticity domain $\mathcal{C}$ can be written as

$$
\mathcal{C}=\{\boldsymbol{\sigma}: f(\boldsymbol{\sigma}) \leq 0\}
$$

for some differentiable, strictly convex function $f$ of the deviatoric stress.

In the case of viscoplasticity, $\partial \phi$ is single-valued and $(2.9)$ can be written more simply as $\dot{\boldsymbol{\varepsilon}}^{p}=\partial \phi(\boldsymbol{\sigma})$. In the case of rate-independent plasticity, the subdifferential $\partial \phi$ is multivalued and Eq. 2.9 corresponds to the normality flow rule

$$
\dot{\boldsymbol{\varepsilon}}^{p}=\lambda \frac{\partial f}{\partial \boldsymbol{\sigma}}(\boldsymbol{\sigma}) \text { with } \lambda \geq 0, f(\boldsymbol{\sigma}) \leq 0, \lambda f(\boldsymbol{\sigma})=0 .
$$

The set of equations (2.1)-(2.4) and (2.7)- $(2.9)$ governs the evolution of the continuum starting from a given initial state. The latter is represented by initial values of the plastic strain and concentration fields. 


\section{Evolution equation for the fields of stress and chemical potential}

\subsection{Weak formulation}

The equations formulated in Sect 2 are now used to derive an evolution equation involving the fields $(\boldsymbol{\sigma}, \mu)$ only, without reference to the other chemo-mechanical fields of the problem. Such an evolution equation will prove to be very convenient in the later sections. The fields $(\boldsymbol{\sigma}, \mu)$ are assumed to live in a subspace $\mathcal{H}$ of $H(\operatorname{div} ; \Omega) \times H^{1}(\Omega)$. We denote by $\mathcal{K}(\boldsymbol{f}, \boldsymbol{T}, M)$ the set of stress and chemical potential fields that are compatible with data $(\boldsymbol{f}, \boldsymbol{T}, M)$, i.e.

$$
\mathcal{K}(\boldsymbol{f}, \boldsymbol{T}, M)=\left\{(\boldsymbol{\sigma}, \mu) \in \mathcal{H}: \operatorname{div} \boldsymbol{\sigma}+\boldsymbol{f}=0 \text { in } \Omega, \boldsymbol{\sigma} \cdot \boldsymbol{n}=\boldsymbol{T} \text { on } \Gamma_{T}, \mu=M \text { on } \Gamma_{\mu}\right\} .
$$

The Euclidean scalar product in $\mathbb{R}_{s}^{3 \times 3} \times \mathbb{R}$ is denoted by :, i.e.

$$
(\boldsymbol{\sigma}, \mu):\left(\boldsymbol{\sigma}^{\prime}, \mu^{\prime}\right)=\boldsymbol{\sigma}: \boldsymbol{\sigma}^{\prime}+\mu \mu^{\prime} .
$$

Observe that the free energy 2.5 can be put in the form

$$
w\left(\varepsilon, \varepsilon^{p}, c\right)=\frac{1}{2}\left(\varepsilon-\varepsilon^{p}, c\right): \mathbb{M}:\left(\varepsilon-\varepsilon^{p}, c\right)+c \mu_{0}
$$

where $\mathbb{M}$ is the symmetric fourth-order tensor determined by the relations

$$
\mathbb{M}:\left(\varepsilon-\varepsilon^{p}, c\right)=\left(\mathbb{L}:\left(\varepsilon-\varepsilon^{p}\right)+c \boldsymbol{A}, k c+\boldsymbol{A}:\left(\varepsilon-\varepsilon^{p}\right)\right) .
$$

for any $\varepsilon-\varepsilon^{p}$ and $c$. Under the requirement (2.6), the tensor $\mathbb{M}$ is symmetric positive definite. Hence the space $\mathcal{H}$ of stress and chemical potential fields is a Hilbert space for the scalar product $\langle\cdot, \cdot\rangle$ defined by

$$
\left\langle(\boldsymbol{\sigma}, \mu),\left(\boldsymbol{\sigma}^{\prime}, \mu^{\prime}\right)\right\rangle=\int_{\Omega}(\boldsymbol{\sigma}, \mu): \mathbb{M}^{-1}:\left(\boldsymbol{\sigma}^{\prime}, \mu^{\prime}\right) d \omega
$$

for any $(\boldsymbol{\sigma}, \mu)$ and $\left(\boldsymbol{\sigma}^{\prime}, \mu^{\prime}\right)$ in $\mathcal{H}$. It can be calculated from $(3.1)$ that

$$
(\boldsymbol{\sigma}, \mu): \mathbb{M}^{-1}:\left(\boldsymbol{\sigma}^{\prime}, \mu^{\prime}\right)=\boldsymbol{\sigma}: \mathbb{L}^{-1}: \boldsymbol{\sigma}^{\prime}+\frac{1}{k^{\prime}}\left((\boldsymbol{B}: \boldsymbol{\sigma})\left(\boldsymbol{B}: \boldsymbol{\sigma}^{\prime}\right)+\mu \mu^{\prime}-\mu^{\prime} \boldsymbol{B}: \boldsymbol{\sigma}-\mu \boldsymbol{B}: \boldsymbol{\sigma}^{\prime}\right)
$$

where $\boldsymbol{B}=\boldsymbol{A}: \mathbb{L}^{-1}$ and $k^{\prime}=k-\boldsymbol{A}: \mathbb{L}^{-1}: \boldsymbol{A}$. Note that the last two terms in 3.3 introduce a coupling between the stress and the chemical potential fields.

Let $\left(\boldsymbol{\sigma}, \mu, \boldsymbol{u}, c, \boldsymbol{J}, \boldsymbol{\varepsilon}^{p}\right)$ be a solution of the evolution problem for the continuum, as detailed in Sect. 2, We necessarily have

$$
(\boldsymbol{\sigma}, \mu) \in \mathcal{K}(\boldsymbol{f}, \boldsymbol{T}, M) .
$$

Consider now an arbitrary $(\boldsymbol{\rho}, \nu)$ in $\mathcal{K}(0,0,0)$ so that

$$
\operatorname{div} \boldsymbol{\rho}=0 \text { in } \Omega, \boldsymbol{\rho} \cdot \boldsymbol{n}=0 \text { on } \Gamma_{T}, \nu=0 \text { on } \Gamma_{\mu} .
$$


The set $\mathcal{K}(0,0,0)$ is more simply denoted by $\mathcal{K}_{0}$ in the following. Noting from (2.7) and (3.1) that $\left(\dot{\boldsymbol{\varepsilon}}-\dot{\boldsymbol{\varepsilon}}^{p}, \dot{c}\right)=\mathbb{M}^{-1}:(\dot{\boldsymbol{\sigma}}, \dot{\mu})$, we have

$$
\langle(\dot{\boldsymbol{\sigma}}, \dot{\mu}),(\boldsymbol{\rho}, \nu)\rangle=\int_{\Omega}\left(\dot{\boldsymbol{\varepsilon}}-\dot{\boldsymbol{\varepsilon}}^{p}\right): \boldsymbol{\rho}+\dot{c} \nu d \omega .
$$

Let us examine the right hand side of (3.5). Integrating by parts and using the mechanical boundary conditions (2.4) as well as the conditions (3.4), we find

$$
\int_{\Omega} \dot{\varepsilon}: \boldsymbol{\rho} d \omega=\int_{\Gamma_{u}} \dot{\boldsymbol{U}} \cdot \boldsymbol{\rho} \cdot \boldsymbol{n} d S
$$

Using the diffusion equation (2.1) and integrating by parts, we have

$$
\int_{\Omega} \dot{c} \nu d \omega=-\int_{\Omega} \nu \operatorname{div} \boldsymbol{\jmath} d \omega=\int_{\Omega} \boldsymbol{\jmath} \cdot \nabla \nu d \omega-\int_{\partial \Omega} \boldsymbol{n} \cdot \boldsymbol{\jmath} \nu d S .
$$

Further using the law of conduction (2.8) as well as the boundary conditions (2.2) and (3.4) yields

$$
\int_{\Omega} \dot{c} \nu d \omega=-\int_{\Omega} \partial \psi(\nabla \mu) \cdot \nabla \nu d \omega-\int_{\Gamma_{J}} J \nu d S
$$

Replacing (3.6) and (3.7) in (3.5), we can finally see that $(\sigma, \mu)$ satisfies

$$
\begin{aligned}
& (\boldsymbol{\sigma}, \mu) \in \mathcal{K}(\boldsymbol{f}, \boldsymbol{T}, M) \\
& \langle(\dot{\boldsymbol{\sigma}}, \dot{\mu}),(\boldsymbol{\rho}, \nu)\rangle=-\int_{\Omega}\left(\dot{\boldsymbol{\varepsilon}}^{p}: \boldsymbol{\rho}+\partial \psi(\nabla \mu) \cdot \nabla \nu\right) d \omega+\int_{\Gamma_{u}} \dot{\boldsymbol{U}} \cdot \boldsymbol{\rho} \cdot \boldsymbol{n} d S-\int_{\Gamma_{J}} J \nu d S \\
& \forall(\boldsymbol{\rho}, \nu) \in \mathcal{K}_{0} .
\end{aligned}
$$

In (3.8), no reference is made to the displacement nor the concentration. Since $\dot{\boldsymbol{\varepsilon}}^{p}$ is directly related to the stress via (2.9), Eq. (3.8) can be interpreted as an evolution equation for the stress and chemical potential fields $(\sigma, \mu)$. We emphasize that the fields $\boldsymbol{\sigma}$ and $\mu$ in $(3.8)$ are coupled implicitly through the scalar product in $(3.2)$ and $(3.3)$. In the next section, we establish some properties of solutions to Eq. (3.8). Those properties will play a crucial role in Sect. 4 when studying the large-time behavior.

\subsection{Contraction properties}

The compact notation $\boldsymbol{\Sigma}=(\boldsymbol{\sigma}, \mu)$ is used in the following. The norm associated to the scalar product $\langle\cdot, \cdot\rangle$ in $(3.2)$ is denoted by $\|\cdot\|$, i.e.

$$
\|\boldsymbol{\Sigma}\|^{2}=\int_{\Omega} \boldsymbol{\Sigma}: \mathbb{M}^{-1}: \Sigma d \omega
$$

We show that the evolution equation (3.8) enjoys the two following properties: 
Property 1. Let $\boldsymbol{\Sigma}$ and $\boldsymbol{\Sigma}^{\prime}$ be two solutions of (3.8). Then

$$
\left\|\boldsymbol{\Sigma}\left(t_{2}\right)-\boldsymbol{\Sigma}^{\prime}\left(t_{2}\right)\right\| \leq\left\|\boldsymbol{\Sigma}\left(t_{1}\right)-\boldsymbol{\Sigma}^{\prime}\left(t_{1}\right)\right\| \text { for all } t_{1}<t_{2} .
$$

Property 2. Let $\boldsymbol{\Sigma}=(\boldsymbol{\sigma}, \mu)$ and $\boldsymbol{\Sigma}^{\prime}=\left(\boldsymbol{\sigma}^{\prime}, \mu^{\prime}\right)$ be two solutions of (3.8) such that $\| \boldsymbol{\Sigma}(t)-$ $\boldsymbol{\Sigma}^{\prime}(t)\|=\| \boldsymbol{\Sigma}\left(t_{1}\right)-\boldsymbol{\Sigma}^{\prime}\left(t_{1}\right) \|$ for all $t_{1} \leq t$. Then there exists a time-independent stress field $\boldsymbol{\rho}$ and a constant $\eta$ such that $(\boldsymbol{\rho}, \eta) \in \mathcal{K}_{0}$ and

$$
\boldsymbol{\sigma}(\boldsymbol{x}, t)-\boldsymbol{\sigma}^{\prime}(\boldsymbol{x}, t)=\boldsymbol{\rho}(\boldsymbol{x}), \mu(\boldsymbol{x}, t)-\mu^{\prime}(\boldsymbol{x}, t)=\eta \text { for } t_{1} \leq t .
$$

In the case of viscoplasticity, we have in addition

$$
\dot{\boldsymbol{\varepsilon}}^{p}(\boldsymbol{x}, t)=\dot{\boldsymbol{\varepsilon}}^{p \prime}(\boldsymbol{x}, t) \text { for } t_{1} \leq t .
$$

The proof of property 1 and 2 is reported in Appendix A and Appendix B, respectively. We emphasize that both properties 1 and 2 crucially rely on the choice $(3.9)$ for the norm on stress and chemical potential fields. Property 1 means that the distance between two solutions of (3.8) is decreasing with time. This notably implies that the solution of $(3.8)$ taking a prescribed value at $t=0$ is unique. Property 2 essentially means that if the distance between two solutions $\boldsymbol{\Sigma}(t)$ and $\boldsymbol{\Sigma}^{\prime}(t)$ is time-independent, then necessarily $\boldsymbol{\Sigma}-$ $\boldsymbol{\Sigma}^{\prime}=\left(\boldsymbol{\sigma}-\boldsymbol{\sigma}^{\prime}, \mu-\mu^{\prime}\right)$ is time-independent. This generalizes a result established by Halphen (1978) in plasticity with no diffusion. Furthermore, property 2 states that $\mu-\mu^{\prime}$ is also space-independent and hence equal to a constant $\eta$. It can observed that the constant $\eta$ is necessarily equal to 0 if $\Gamma_{\mu} \neq \emptyset$, i.e. if the chemical potential is imposed on a part of the boundary. By contrast, there is no need for the stress field $\rho$ in $(3.10)$ to be spaceindependent, even if tractions are imposed all over the boundary.

\section{Large-time behavior under cyclic chemo-mechanical loadings}

From now on we assume that the functions $(M, J, \boldsymbol{f}, \boldsymbol{T}, \boldsymbol{U})$ defining the chemo-mechanical loading history are all periodic in time with the same period $T$ (such functions are referred to as $T$-periodic). Our objective in this section is to study the behavior of solutions to (3.8) as time tends to infinity.

\subsection{Periodic solutions}

$T$-periodic solutions to (3.8) will play an important role in the subsequent developments. Although there is not uniqueness, $T$-periodic solutions to (3.8) are quite similar to one another, as demonstrated by the following 
Property 3. Let $\boldsymbol{\Sigma}=(\boldsymbol{\sigma}, \mu)$ and $\boldsymbol{\Sigma}^{\prime}=\left(\boldsymbol{\sigma}^{\prime}, \mu^{\prime}\right)$ be two T-periodic solutions of (3.8). Then there exists a time-independent stress field $\boldsymbol{\rho}$ and a constant $\eta$ such that $(\boldsymbol{\rho}, \eta) \in \mathcal{K}_{0}$ and

$$
\boldsymbol{\sigma}(\boldsymbol{x}, t)-\boldsymbol{\sigma}^{\prime}(\boldsymbol{x}, t)=\boldsymbol{\rho}(\boldsymbol{x}), \mu(\boldsymbol{x}, t)-\mu^{\prime}(\boldsymbol{x}, t)=\eta .
$$

In the case of viscoplasticity, we have in addition

$$
\dot{\boldsymbol{\varepsilon}}^{p}(\boldsymbol{x}, t)=\dot{\boldsymbol{\varepsilon}}^{p \prime}(\boldsymbol{x}, t)
$$

Let indeed $\boldsymbol{\Sigma}=(\boldsymbol{\sigma}, \mu)$ and $\boldsymbol{\Sigma}^{\prime}=\left(\boldsymbol{\sigma}^{\prime}, \mu^{\prime}\right)$ be two $T$-periodic solutions of (3.8). Property 1 yields

$$
\left\|\boldsymbol{\Sigma}(T)-\boldsymbol{\Sigma}^{\prime}(T)\right\| \leq\left\|\boldsymbol{\Sigma}(t)-\boldsymbol{\Sigma}^{\prime}(t)\right\| \leq\left\|\boldsymbol{\Sigma}(0)-\boldsymbol{\Sigma}^{\prime}(0)\right\|
$$

for all $t \in[0, T]$. By $T$-periodicity of $\boldsymbol{\Sigma}$ and $\boldsymbol{\Sigma}^{\prime}$, we have $\left\|\boldsymbol{\Sigma}(T)-\boldsymbol{\Sigma}^{\prime}(T)\right\|=\left\|\boldsymbol{\Sigma}(0)-\boldsymbol{\Sigma}^{\prime}(0)\right\|$. It follows that 4.1 holds as an equality, i.e. that $\left\|\boldsymbol{\Sigma}(t)-\boldsymbol{\Sigma}^{\prime}(t)\right\|$ is independent of $t$. Applying property 2 gives the desired result.

\subsection{Convergence to a periodic solution}

We are now in a position to prove the main result of this section:

Property 4. Assume that there exists a T-periodic solution to (3.8) and that the dimension of $\mathcal{H}$ is finite. Then every solution of (3.8) converges to a $T$-periodic solution as $t \rightarrow \infty$

A convenient way to prove the claim is to use Opial's lemma (Opial, 1967), which is a general result in Hilbert spaces that has proved to be very useful in several studies related to large time convergence (see e.g Baillon and Haraux (1977); Johnson et al. (1978); Wesfreid (1981)). In the present context, Opial's lemma reads as follows:

Let $F$ be a nonempty subset of $\mathcal{H}$ and $\left\{u_{n}\right\}$ a sequence in $\mathcal{H}$ such that :

(i) $\left\{\left\|u_{n}-f\right\|\right\}$ converges for all $f \in F$,

(ii) the limit of every (weakly) convergent subsequence of $\left\{u_{n}\right\}$ is in $F$.

Then the whole sequence $\left\{u_{n}\right\}$ (weakly) converges to a point of $F$.

The proof of Opial's lemma is recalled in Appendix C for the sake of completeness. In the following we will only consider the simplified situation where the dimension of $\mathcal{H}$ is finite, so that weak and strong convergences coincide. Considering an arbitrary solution $\boldsymbol{\Sigma}(t)$ of (3.8), we will apply Opial's lemma to the sequence $\left\{u_{n}\right\}=\{\boldsymbol{\Sigma}(n T)\}$ and to the set $F$ formed by the initial values of $T$-periodic solutions to $(3.8)$, i.e.

$$
F=\{\tilde{\boldsymbol{\Sigma}}(0): t \mapsto \tilde{\boldsymbol{\Sigma}}(t) \text { is a } T \text {-periodic solution of }(3.8)\}
$$


In order to check assumption $(i)$ in Opial's lemma, consider $f \in F$ and let $\boldsymbol{\Sigma}^{\prime}$ be a $T$-periodic solution of (3.8) such that $f=\boldsymbol{\Sigma}^{\prime}(0)$. By periodicity, we have

$$
\boldsymbol{\Sigma}^{\prime}(n T)=f
$$

for all $n \in \mathbb{N}$. We know from property 1 that

$$
\left\|\boldsymbol{\Sigma}((n+1) T)-\boldsymbol{\Sigma}^{\prime}((n+1) T)\right\| \leq\left\|\boldsymbol{\Sigma}(n T)-\boldsymbol{\Sigma}^{\prime}(n T)\right\|,
$$

i.e. that $\left\|u_{n+1}-f\right\| \leq\left\|u_{n}-f\right\|$. The positive sequence $\left\{\left\|u_{n}-f\right\|\right\}$ is thus decreasing. It follows that

$$
\|\Sigma(n T)-f\| \underset{n \rightarrow \infty}{\longrightarrow} A
$$

for some constant $A$. The assumption $(i)$ in Opial's lemma is thus verified. To check the assumption (ii), consider a converging subsequence $\left\{u_{n_{k}}\right\}$ of $\left\{u_{n}\right\}$ and denote its limit by $g$. Let $\boldsymbol{\Sigma}^{*}(t)$ be the solution of (3.8) for the initial condition $\boldsymbol{\Sigma}^{*}(0)=g$. We wish to show that $g \in F$, i.e. that $\boldsymbol{\Sigma}^{*}(t)$ is $T$-periodic. Considering a fixed $t$ in the interval $[0, T]$ and using property 1 with $\boldsymbol{\Sigma}$ and $\boldsymbol{\Sigma}^{*}$, we have

$$
\left\|\boldsymbol{\Sigma}\left(n_{k} T+t\right)-\boldsymbol{\Sigma}^{*}(t)\right\| \leq\left\|\boldsymbol{\Sigma}\left(n_{k} T\right)-\boldsymbol{\Sigma}^{*}(0)\right\|=\left\|u_{n_{k}}-g\right\| \underset{k \rightarrow \infty}{\longrightarrow} 0 .
$$

Thus

$$
\boldsymbol{\Sigma}\left(n_{k} T+t\right) \underset{k \rightarrow \infty}{\longrightarrow} \boldsymbol{\Sigma}^{*}(t)
$$

for any given $t$ in $[0, T]$. Besides we have

$$
n_{k} T \leq n_{k} T+t \leq n_{k+1} T
$$

hence, using property 1 with $\boldsymbol{\Sigma}$ and $\boldsymbol{\Sigma}^{\prime}$,

$$
\left\|\boldsymbol{\Sigma}\left(n_{k+1} T\right)-\boldsymbol{\Sigma}^{\prime}\left(n_{k+1} T\right)\right\| \leq\left\|\boldsymbol{\Sigma}\left(n_{k} T+t\right)-\boldsymbol{\Sigma}^{\prime}(t)\right\| \leq\left\|\boldsymbol{\Sigma}\left(n_{k} T\right)-\boldsymbol{\Sigma}^{\prime}\left(n_{k} T\right)\right\|
$$

where the $T$-periodicity of $\boldsymbol{\Sigma}^{\prime}$ has been used. Using (4.2), Eq. 4.5) can be rewritten more simply as

$$
\left\|\boldsymbol{\Sigma}\left(n_{k+1} T\right)-f\right\| \leq\left\|\boldsymbol{\Sigma}\left(n_{k} T+t\right)-\boldsymbol{\Sigma}^{\prime}(t)\right\| \leq\left\|\boldsymbol{\Sigma}\left(n_{k} T\right)-f\right\| .
$$

By (4.3), both the left and the right terms in (4.6) converge to $A$ as $k \rightarrow \infty$. It follows that

$$
\left\|\boldsymbol{\Sigma}\left(n_{k} T+t\right)-\boldsymbol{\Sigma}^{\prime}(t)\right\| \underset{k \rightarrow \infty}{\longrightarrow} A .
$$

Combining 4.4 and (4.7), it can be seen that

$$
\left\|\boldsymbol{\Sigma}^{*}(t)-\boldsymbol{\Sigma}^{\prime}(t)\right\|=A
$$

for all $t$ in $[0, T]$. By property 2 we can conclude that $\boldsymbol{\Sigma}^{*}(t)-\boldsymbol{\Sigma}^{\prime}(t)$ is time-independent. Hence $\boldsymbol{\Sigma}^{*}(t)$ is $T$-periodic, i.e. $\boldsymbol{\Sigma}^{*}(0)=g \in F$. The assumption (ii) is thus verified. Applying Opial's lemma gives the desired result: For any solution $\boldsymbol{\Sigma}(t)$ of (3.8), there exists a $T$-periodic solution $\boldsymbol{\Sigma}^{*}(t)$ such that $\boldsymbol{\Sigma}(n T) \rightarrow \boldsymbol{\Sigma}^{*}(0)$ as $n \rightarrow \infty$. By property 1 , it follows that $\boldsymbol{\Sigma}(t)-\boldsymbol{\Sigma}^{*}(t) \rightarrow 0$ as $n \rightarrow \infty$. 


\subsection{Consequences}

Let us collect the results we have established so far. Under the assumptions that there exists a $T$-periodic solution to (3.8) and that the dimension of $\mathcal{H}$ is finite, property 4 shows that the stress and the chemical potential fields always become $T$-periodic in the large time limit, regardless of the initial state. Noting from 3.1$)$ that $\left(\varepsilon-\varepsilon^{p}, c\right)=\mathbb{M}^{-1}:\left(\sigma, \mu-\mu_{0}\right)$, it follows that the elastic strain $\varepsilon-\varepsilon^{p}$ and the concentration $c$ also become $T$-periodic in the large time limit.

Since there is not necessarily uniqueness of $T$-periodic solutions to (3.8), the asymptotic $T$-periodic response $\left(\boldsymbol{\sigma}_{\infty}, \mu_{\infty}\right)$ depends on the initial state considered. However, the property 3 shows that some features of the asymptotic response are independent of the initial state. This is notably the case of the stress rate $\dot{\boldsymbol{\sigma}}_{\infty}$, the flux $\boldsymbol{J}_{\infty}(\boldsymbol{x}, t)$ and - possibly up to a constant if $\Gamma_{\mu}=\emptyset$ - of the chemical potential $\mu_{\infty}(\boldsymbol{x}, t)$. Thus diffusion reaches a cyclic steady state which is largely independent of any residual stress and residual concentration of guest atoms in the initial state. For lithium-ion batteries, diffusion-related quantities are directly related to operational electrical quantities. More precisely, the flux $\boldsymbol{J}$ is related to the charge and the chemical potential $\mu$ is related to the voltage. Therefore, the charge-voltage response - which is of primary concern in lithium-ion batteries - reaches a cyclic steady state that is essentially independent of the initial conditions (some examples will be given in Sect. 6, see Fig. 14).

For a viscoplastic behavior, property 4 also shows that the plastic strain rate converges to a $T$-periodic response $\dot{\boldsymbol{\varepsilon}}_{\infty}^{p}$. The situation where $\dot{\boldsymbol{\varepsilon}}_{\infty}^{p}=0$ corresponds to elastic shakedown. When $\dot{\varepsilon}_{\infty}^{p} \neq 0$, only two cases can occur depending on the value of $\int_{0}^{T} \dot{\varepsilon}_{\infty}^{p} d t$. Indeed, if $\int_{0}^{T} \dot{\boldsymbol{\varepsilon}}_{\infty}^{p} d t=0$, then $\dot{\boldsymbol{\varepsilon}}_{\infty}^{p}(t+T)=\dot{\boldsymbol{\varepsilon}}_{\infty}^{p}(t)$ i.e. cyclic plasticity occurs. Otherwise $\left(\int_{0}^{T} \dot{\boldsymbol{\varepsilon}}_{\infty}^{p} d t \neq 0\right)$, there is plastic strain accumulation with a constant increment over each cycle, i.e. ratcheting occurs. Whatever the geometry and the loading, the plastic behavior necessarily falls in one of those three categories (elastic shakedown, cyclic plasticity, ratcheting) - there is no other possible regime. Moreover, as a consequence of the property 3, the plastic strain rate $\dot{\boldsymbol{\varepsilon}}_{\infty}^{p}$ in the cyclic steady state is independent of the initial state. Therefore, for a given geometry, the type of plastic behavior depends only of the loading. Those theoretical results justify establishing some maps in the space of load parameters, in the spirit of Bree diagrams (Bree, 1967) : For each point in the map are recorded corresponding path-independent properties such as the type of plastic behavior, the plastic dissipation $\int_{0}^{T} \boldsymbol{\sigma}_{\infty}: \dot{\boldsymbol{\varepsilon}}_{\infty}^{p} d t$ and, for lithium-ion batteries, the charge-voltage response in the steady state.

Such a map has been reported by Brassart et al. (2013) for a spherical particle of electrode material under cyclic lithiation. For each considered values of the loading parameters, the cyclic steady state was estimated using incremental analysis over a sufficiently large number of cycles. The case of elastic shakedown was identified as the most favorable for the electromechanical efficiency because the hysteresis in the charge-voltage response was found to be reduced. It can be added that elastic shakedown is also expected to be fa- 
vorable for the mechanical fatigue behavior (Constantinescu et al. 2003). Building on such arguments, elastic shakedown appears as the most desirable regime for lithium-ion batteries. Using properties 3 and 4 , it is possible to find the set of loading parameters for which elastic shakedown occurs, without resorting to incremental analysis of the coupled plasticity-diffusion problem. That point is the focus of the next section.

\section{Elastic shakedown under cyclic chemo-mechanical loading}

\subsection{Conditions for elastic shakedown}

Let us consider the purely elasto-diffusive problem obtained by discarding plastic flow in the original problem. That fictitious problem is defined by the very same equations as in Sect.2, except that the dissipation potential $\phi$ is set to 0 . As a particular case of the developments presented in Sect. 3, the stress and chemical potential fields $(\tilde{\boldsymbol{\sigma}}, \tilde{\mu})$ of the purely elasto-diffusive problem satisfy

$$
\begin{aligned}
& (\tilde{\boldsymbol{\sigma}}, \tilde{\mu}) \in \mathcal{K}(\boldsymbol{f}, \boldsymbol{T}, M), \\
& \langle(\dot{\tilde{\boldsymbol{\sigma}}}, \dot{\tilde{\mu}}),(\boldsymbol{\rho}, \nu)\rangle=-\int_{\Omega} \partial \psi(\nabla \tilde{\mu}) \cdot \nabla \nu d \omega+\int_{\Gamma_{u}} \dot{\boldsymbol{U}} \cdot \boldsymbol{\rho} \cdot \boldsymbol{n} d S-\int_{\Gamma_{J}} J \nu d S \\
& \forall(\boldsymbol{\rho}, \nu) \in \mathcal{K}_{0} .
\end{aligned}
$$

Uniqueness of the solution of $(5.1)$ is obtained if one further specifies the value of $(\tilde{\boldsymbol{\sigma}}, \tilde{\mu})$ at time $t=0$. Eq. (5.1) gives the stress and chemical potential fields that would reign in the body if plasticity was ignored.

Eq. (5.1) is instrumental for formulating conditions under which elastic shakedown occurs for the original problem coupling plasticity with diffusion, as is now explained. In all the reasoning that follows, we consider the $T$-periodic asymptotic response $\left(\boldsymbol{\sigma}_{\infty}, \mu_{\infty}\right)$ of the coupled plasticity-diffusion problem $(3.8)$ for some (unspecified) initial state and we denote by $\dot{\varepsilon}_{\infty}^{p}$ the corresponding plastic strain rate. Elastic shakedown corresponds to the situation where $\dot{\boldsymbol{\varepsilon}}_{\infty}^{p}(\boldsymbol{x}, t)=0$, in which case the evolution equation (3.8) yields

$$
\left\langle\left(\dot{\boldsymbol{\sigma}}_{\infty}, \dot{\mu}_{\infty}\right),(\boldsymbol{\rho}, \nu)\right\rangle=-\int_{\Omega} \partial \psi\left(\nabla \mu_{\infty}\right) \cdot \nabla \nu d \omega+\int_{\Gamma_{u}} \dot{\boldsymbol{U}} \cdot \boldsymbol{\rho} \cdot \boldsymbol{n} d S-\int_{\Gamma_{J}} J \nu d S
$$

for all $(\boldsymbol{\rho}, \nu) \in \mathcal{K}_{0}$. In the case of viscoplasticity, the condition $\dot{\boldsymbol{\varepsilon}}_{\infty}^{p}(\boldsymbol{x}, t)=0$ also implies that

$$
\boldsymbol{\sigma}_{\infty}(\boldsymbol{x}, t) \in \mathcal{C} .
$$

Comparing (5.2) with (5.1) shows that a necessary condition for shakedown is given by the following

Shakedown condition 1 (viscoplasticity). There exists a T-periodic solution $(\tilde{\boldsymbol{\sigma}}, \tilde{\mu})$ to the purely elasto-diffusive equation (5.1) that satisfies the condition $\tilde{\boldsymbol{\sigma}} \in \mathcal{C}$. 
In the case of viscoplasticity, the condition 1 is also sufficient for shakedown to occur. Let indeed $(\tilde{\boldsymbol{\sigma}}, \tilde{\mu})$ satisfying condition 1 . In viscoplasticity, the relation $\tilde{\boldsymbol{\sigma}} \in \mathcal{C}$ implies that $\partial \phi(\tilde{\boldsymbol{\sigma}})=0$. Hence $(\tilde{\boldsymbol{\sigma}}, \tilde{\mu})$ is also a $T$-periodic solution of $(3.8)$ and the corresponding plastic strain $\tilde{\boldsymbol{\varepsilon}}^{p}$ vanishes everywhere. Property 3 implies that $\dot{\boldsymbol{\varepsilon}}_{\infty}^{p}=d \tilde{\boldsymbol{\varepsilon}}^{p} / d t=0$.

In the case of rate-independent plasticity, similar arguments show that the condition 1 is necessary for shakedown to occur. A sufficient condition is provided by the more restrictive

Shakedown condition 2 (rate-independent plasticity). There exists a T-periodic solution $(\tilde{\boldsymbol{\sigma}}, \tilde{\mu})$ to the purely elasto-diffusive equation (5.1) such that $\tilde{\boldsymbol{\sigma}}$ is in the interior of $\mathcal{C}$.

Let indeed be $(\tilde{\boldsymbol{\sigma}}, \tilde{\mu})$ satisfying condition 2 . Note that $(\tilde{\boldsymbol{\sigma}}, \tilde{\mu})$ is also a solution of the elastic-plastic problem (3.8). By applying the property 3 to the two $T$-periodic solutions $\boldsymbol{\Sigma}_{\infty}=(\tilde{\boldsymbol{\sigma}}, \tilde{\mu})$ and $\tilde{\boldsymbol{\Sigma}}=\left(\boldsymbol{\sigma}_{\infty}, \mu_{\infty}\right)$, we obtain that $\left\|\boldsymbol{\Sigma}_{\infty}(t)-\tilde{\boldsymbol{\Sigma}}(t)\right\|$ is time-independent. Reproducing the arguments used in Appendix B for proving property 2, we obtain

$$
\left(\boldsymbol{\sigma}_{\infty}-\tilde{\boldsymbol{\sigma}}\right): \dot{\boldsymbol{\varepsilon}}_{\infty}^{p}=0
$$

Since the elasticity domain $\mathcal{C}$ is strictly convex in the deviatoric space and $\tilde{\boldsymbol{\sigma}}$ is in the interior of $\mathcal{C}$, the condition $(5.3)$ can only be realized if $\dot{\varepsilon}_{\infty}^{p}=0$. Suppose indeed for the sake of contradiction that $\dot{\varepsilon}_{\infty}^{p} \neq 0$. By (2.11) we have

$$
f\left(\boldsymbol{\sigma}_{\infty}\right)=0, \quad \dot{\boldsymbol{\varepsilon}}_{\infty}^{p}=\lambda \frac{\partial f}{\partial \boldsymbol{\sigma}}\left(\boldsymbol{\sigma}_{\infty}\right)
$$

where $\lambda>0$. Since $\tilde{\boldsymbol{\sigma}}$ is in the interior of $\mathcal{C}$, we have

$$
f(\tilde{\boldsymbol{\sigma}})<0 .
$$

The strict convexity of $f$ implies that

$$
f(\tilde{\boldsymbol{\sigma}})-f\left(\boldsymbol{\sigma}_{\infty}\right)>\left(\tilde{\boldsymbol{\sigma}}-\boldsymbol{\sigma}_{\infty}\right): \frac{\partial f}{\partial \boldsymbol{\sigma}}\left(\boldsymbol{\sigma}_{\infty}\right) .
$$

Multiplying the above inequality by $\lambda$ and using (5.3) as well as (5.4), we obtain that $f(\tilde{\boldsymbol{\sigma}})>0$ which is contradiction with Eq. (5.5).

In view of the shakedown conditions 1 and 2 , the issue of characterizing $T$-periodic solutions to the elasto-diffusive equation (5.1) is essential for determining whether elastic shakedown occurs or not. That issue is addressed in more detail in the next section, with special emphasis on the case of linear diffusion. 


\subsection{Periodic solutions in elasto-diffusion}

Let $\left(\boldsymbol{\sigma}^{E}, \mu^{E}\right)$ be a particular $T$-periodic solution to the elasto-diffusive equation (5.1). Applying the property 3 , any $T$-periodic solution $(\tilde{\boldsymbol{\sigma}}, \tilde{\mu})$ to 5.1 can be written as

$$
\tilde{\boldsymbol{\sigma}}(\boldsymbol{x}, t)=\boldsymbol{\sigma}^{E}(\boldsymbol{x}, t)+\tilde{\boldsymbol{\rho}}(\boldsymbol{x}), \tilde{\mu}(\boldsymbol{x}, t)=\mu^{E}(\boldsymbol{x}, t)+\tilde{\eta}
$$

for some time-independent stress field $\tilde{\boldsymbol{\rho}}$ and some constant $\tilde{\eta}$ such that $(\tilde{\boldsymbol{\rho}}, \tilde{\eta}) \in \mathcal{K}_{0}$. Conversely, for any $(\tilde{\boldsymbol{\rho}}, \tilde{\eta}) \in \mathcal{K}_{0}$, it can easily be verified that the field $(\tilde{\boldsymbol{\sigma}}, \tilde{\mu})$ given $(5.6)$ is a $T$-periodic solution of (5.1). Hence, the whole family of $T$-periodic solutions to the elasto-diffusive equation $(5.1)$ is

$$
\left\{\left(\boldsymbol{\sigma}^{E}+\tilde{\boldsymbol{\rho}}, \mu^{E}+\tilde{\eta}\right):(\tilde{\boldsymbol{\rho}}, \tilde{\eta}) \in \mathcal{K}_{0} ; \tilde{\boldsymbol{\rho}} \text { time-independent; } \tilde{\eta} \text { constant }\right\} .
$$

Further progress can be made if the diffusion is linear, i.e. obeys the Fick's law $\boldsymbol{J}=$ $-(D / k) \nabla \mu$ where $D$ is the diffusion parameter, assumed to be independent of $\mu$. In such case, the pure elasto-diffusion problem reads as

$$
\begin{gathered}
(\boldsymbol{\sigma}, \mu) \in \mathcal{K}(\boldsymbol{f}, \boldsymbol{T}, M), \\
(\boldsymbol{u}, \boldsymbol{\jmath}) \in \mathcal{K}^{\prime}(\boldsymbol{U}, J), \\
(\boldsymbol{\sigma}, \mu)=\mathbb{M}:(\boldsymbol{\varepsilon}(\boldsymbol{u}), c)+\left(0, \mu_{0}\right), \\
\boldsymbol{\jmath}=-(D / k) \nabla \mu \\
\dot{c}+\operatorname{div} \boldsymbol{\jmath}=0
\end{gathered}
$$

where $\varepsilon(\boldsymbol{u})=\left(\nabla \boldsymbol{u}+\nabla^{T} \boldsymbol{u}\right) / 2$ and $\mathcal{K}^{\prime}(\boldsymbol{U}, J)$ is the set of displacement and flux fields compatible with data $(\boldsymbol{U}, J)$, i.e.

$$
\mathcal{K}^{\prime}(\boldsymbol{U}, J)=\left\{(\boldsymbol{u}, \boldsymbol{\jmath}): \boldsymbol{u}=\boldsymbol{U} \text { on } \Gamma_{u}, \boldsymbol{\jmath} \cdot \boldsymbol{n}=J \text { on } \Gamma_{J}\right\} .
$$

A $T$-periodic solution $\left(\boldsymbol{\sigma}^{E}, \mu^{E}, \boldsymbol{u}^{E}, c^{E}\right)$ to $(5.8)$ can be constructed using Fourier expansion, writing

$$
\begin{aligned}
& \boldsymbol{\sigma}^{E}(\boldsymbol{x}, t)=\sum_{n=-\infty}^{+\infty} \hat{\boldsymbol{\sigma}}_{n}(\boldsymbol{x}) e^{i n \omega t}, \quad \mu^{E}(\boldsymbol{x}, t)=\sum_{n=-\infty}^{+\infty} \hat{\mu}_{n}(\boldsymbol{x}) e^{i n \omega t} \\
& \boldsymbol{u}^{E}(\boldsymbol{x}, t)=\sum_{n=-\infty}^{+\infty} \hat{\boldsymbol{u}}_{n}(\boldsymbol{x}) e^{i n \omega t}, \quad c^{E}(\boldsymbol{x}, t)=\sum_{n=-\infty}^{+\infty} \hat{c}_{n}(\boldsymbol{x}) e^{i n \omega t}
\end{aligned}
$$

where $\omega=2 \pi / T$ and $\left(\hat{\boldsymbol{\sigma}}_{n}, \hat{\mu}_{n}, \hat{\boldsymbol{u}}_{n}, \hat{c}_{n}\right)$ are complex-valued fields defined in $\Omega$. The requirement that $\boldsymbol{\sigma}^{E}$ is real-valued implies that $\hat{\boldsymbol{\sigma}}_{n}$ and $\hat{\boldsymbol{\sigma}}_{-n}$ are complex conjugates (the same remark applies to the other fields in (5.10). Substituting (5.10) in (5.8) leads to the harmonic elasto-diffusion problem

$$
\begin{gathered}
\left(\hat{\boldsymbol{\sigma}}_{n}, \hat{\mu}_{n}\right) \in \mathcal{K}\left(\hat{\boldsymbol{f}}_{n}, \hat{\boldsymbol{T}}_{n}, \hat{M}_{n}\right), \\
\left(\hat{\boldsymbol{u}}_{n}, \hat{\boldsymbol{\jmath}}_{n}\right) \in \mathcal{K}^{\prime}\left(\hat{\boldsymbol{U}}_{n}, \hat{J}_{n}\right), \\
\left(\hat{\boldsymbol{\sigma}}_{n}, \hat{\mu}_{n}\right)=\mathbb{M}:\left(\varepsilon\left(\hat{\boldsymbol{u}}_{n}\right), \hat{c}_{n}\right)+\left(0, \delta_{n 0} \mu_{0}\right), \\
\hat{\boldsymbol{\jmath}}_{n}=-(D / k) \nabla \hat{\mu}_{n}, \\
i n \omega \hat{c}_{n}+\operatorname{div} \hat{\boldsymbol{\jmath}}_{n}=0 ;
\end{gathered}
$$


where $\hat{\boldsymbol{f}}_{n}$ (resp. $\hat{\boldsymbol{T}}_{n}, \hat{M}_{n}, \hat{\boldsymbol{U}}_{n}, \hat{\boldsymbol{J}}_{n}$ ) are the Fourier components of $\boldsymbol{f}$ (resp. $\boldsymbol{T}, M, \boldsymbol{U}, J$ ), i.e. $\hat{\boldsymbol{f}}_{n}=\frac{1}{T} \int_{0}^{T} \boldsymbol{f}(t) e^{-i n \omega t} d t$. The boundary value problem (5.11) is linear and elliptic, i.e. it only involves space variables. For simple geometries, the problem (5.11) can be solved explicitly as will be illustrated in Sect. 6 .

\subsection{Shakedown design in coupled plasticity-diffusion}

The results obtained suggest a general two-step approach for the shakedown design of cyclically loaded bodies in which plasticity is coupled with diffusion:

Step 1. Calculate a $T$-periodic solution $\left(\boldsymbol{\sigma}^{E}, \mu^{E}\right)$ to the purely elasto-diffusive equation (5.1).

Step 2. Using the characterization (5.7), check whether some $(\tilde{\boldsymbol{\sigma}}, \tilde{\mu})$ satisfying the shakedown condition 1 (in viscoplasticity) or 2 (in rate-independent plasticity) can be found. In such case, elastic shakedown occurs regardless of the initial state.

Let us elaborate on Step 2, focusing - to fix ideas- on the shakedown condition 1. In view of (5.7), checking condition 1 amounts to checking if one can find a time-independent stress field $\tilde{\boldsymbol{\rho}}$ verifying

$$
\operatorname{div} \tilde{\boldsymbol{\rho}}=0 \text { in } \Omega, \tilde{\boldsymbol{\rho}} \cdot \boldsymbol{n}=0 \text { on } \Gamma_{T}
$$

and

$$
\boldsymbol{\sigma}^{E}(\boldsymbol{x}, t)+\tilde{\boldsymbol{\rho}}(\boldsymbol{x}) \in \mathcal{C}
$$

for all $\boldsymbol{x}$ and $t \in[0, T]$. An important observation is that the conditions (5.12) and (5.13) are identical to those used in the shakedown design of standard elastic-plastic bodies (i.e. with no diffusion). Consequently, Step 2 can be performed using available methods that have been proposed in standard plasticity (Maier et al., 2003). All the specificities of the coupling with diffusion are thus concentrated in Step 1. For Fick's law, performing Step 1 amounts to solve a sequence of "equilibrium-type" problems as detailed in Sect. 5.2.

In the next section, the suggested two-step approach is applied on a model problem related to lithium-ion batteries.

\section{Application to a lithium-ion battery electrode particle}

We consider the model problem of a cylinder electrode particle with radius $R$, under the assumption of plane strain. Cyclic lithiation-delithiation is achieved by applying a $T$-periodic flux $J(t)$ of lithium on the outer surface $r=R$ (Fig. 1). Body forces are neglected and the outer surface $r=R$ is considered as stress-free.

The constitutive material is taken as isotropic, in which case the free energy in (2.5) specializes as

$$
w\left(\varepsilon, \varepsilon^{p}, c\right)=\frac{1}{2} K(\operatorname{tr} \varepsilon)^{2}+G\left(\varepsilon^{d}-\varepsilon^{p}\right):\left(\varepsilon^{d}-\varepsilon^{p}\right)+\frac{1}{2} k c^{2}+a c \operatorname{tr} \varepsilon+c \mu_{0}
$$




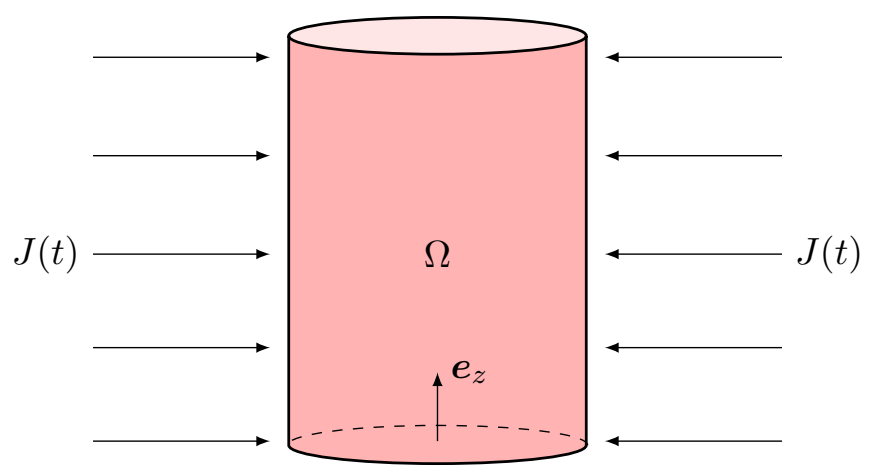

Figure 1: A cylinder particle under cyclic lithiation-delithiation.

where $\varepsilon^{d}$ is the deviatoric strain and $c$ is the concentration of lithium. In 6.1 , the bulk modulus $K$ and the shear modulus $G$ are related to Young's modulus $E$ and Poisson's ratio $\nu$ by the expressions $K=E / 3(1-2 \nu)$ and $G=E / 2(1+\nu)$. The scalar material parameter $a$ in (6.1) accounts for the chemo-mechanical coupling. The constitutive relations (2.7) become

$$
\begin{gathered}
\sigma^{m}=K \operatorname{tr} \varepsilon+a c, \quad s=2 G\left(\varepsilon^{d}-\varepsilon^{p}\right), \\
\mu=\mu_{0}+k c+a \operatorname{tr} \varepsilon,
\end{gathered}
$$

where $\sigma^{m}=(\operatorname{tr} \boldsymbol{\sigma}) / 3$ is the hydrostatic stress and $s$ is the deviatoric stress. In particular, for stress-free states in the elastic regime, Eq. 6.2 gives

$$
\operatorname{tr} \varepsilon=-\frac{a}{K} c
$$

hence the ratio $-a / K$ can be interpreted as the volumetric expansion coefficient associated with the insertion of lithium. Positive (resp. negative) values of $a$ correspond to situations where the insertion of lithium involves a contraction (resp. expansion) of the volume. We can observe that the requirement (2.6) of convexity of the free energy $w$ translates as

$$
k K-a^{2}>0
$$

and therefore does not place any constraint on the sign of $a$.

\subsection{Harmonic loading}

We first consider the harmonic loading

$$
J(t)=-\frac{R \omega}{4} H \sin \omega t
$$

where $\omega=2 \pi / T$ and $H>0$ are given. Lithiation occurs for $0 \leq t \leq T / 2$ and delithiation occurs for $T / 2 \leq t \leq T$. The loading parameter $H$ in (6.4) is directly related to the maximum number of inserted lithium ions. More precisely, for a unit length in the $z$ direction, the maximum number of inserted lithium ions is given by $-2 \pi R \int_{0}^{T / 2} J(t) d t=$ $\pi R^{2} H$ which corresponds to an average concentration equal to $H$. 


\subsubsection{Cyclic steady state in elasto-diffusion}

Let us first consider the case where the constitutive material is purely elastic. For the harmonic loading (6.4), a particular $T$ - periodic solution $\left(\boldsymbol{\sigma}^{E}, \mu^{E}, \boldsymbol{u}^{E}, c^{E}\right)$ to the elastodiffusive problem (5.8) can be sought in the form

$$
\begin{array}{ll}
\boldsymbol{\sigma}^{E}(\boldsymbol{x}, t)=\Re\left(\hat{\boldsymbol{\sigma}}(\boldsymbol{x}) e^{i \omega t}\right), & \mu^{E}(\boldsymbol{x}, t)=\mu_{0}+\Re\left(\hat{\mu}(\boldsymbol{x}) e^{i \omega t}\right), \\
\boldsymbol{u}^{E}(\boldsymbol{x}, t)=\Re\left(\hat{\boldsymbol{u}}(\boldsymbol{x}) e^{i \omega t}\right), & c^{E}(\boldsymbol{x}, t)=\Re\left(\hat{c}(\boldsymbol{x}) e^{i \omega t}\right),
\end{array}
$$

where $(\hat{\boldsymbol{\sigma}}, \hat{\mu}, \hat{\boldsymbol{u}}, \hat{c})$ is the solution of $(5.11)$ with $n=1$ and $\hat{J}_{1}=i R \omega H / 4$. As in Sect. 5.2 . the superscript ${ }^{\wedge}$ is used to denote complex-values quantities. The real and imaginary parts of complex-valued quantities are denoted by $\Re$ and $\Im$, respectively.

In view of the circular symmetry of the problem, we look for a solution in the form

$$
\hat{\boldsymbol{u}}(\boldsymbol{x})=\hat{u}(r) \boldsymbol{e}_{r}, \hat{c}(\boldsymbol{x})=\hat{c}(r)
$$

where $\left(\boldsymbol{e}_{r}, \boldsymbol{e}_{\theta}, \boldsymbol{e}_{z}\right)$ is the local basis for the cylindrical coordinates $(r, \theta, z)$. The constitutive relations 6.2) show that $\hat{\boldsymbol{\sigma}}=\hat{\sigma}_{r} \boldsymbol{e}_{r} \otimes \boldsymbol{e}_{r}+\hat{\sigma}_{\theta} \boldsymbol{e}_{\theta} \otimes \boldsymbol{e}_{\theta}+\hat{\sigma}_{z} \boldsymbol{e}_{z} \otimes \boldsymbol{e}_{z}$ where

$$
\begin{aligned}
& \hat{\sigma}_{r}=\frac{2 G}{3}\left(2 \frac{d \hat{u}}{d r}-\frac{\hat{u}}{r}\right)+K\left(\frac{d \hat{u}}{d r}+\frac{\hat{u}}{r}\right)+a \hat{c}, \\
& \hat{\sigma}_{\theta}=\frac{2 G}{3}\left(2 \frac{\hat{u}}{r}-\frac{d \hat{u}}{d r}\right)+K\left(\frac{d \hat{u}}{d r}+\frac{\hat{u}}{r}\right)+a \hat{c}, \\
& \hat{\sigma}_{z}=-\frac{2 G}{3}\left(\frac{d \hat{u}}{d r}+\frac{\hat{u}}{r}\right)+K\left(\frac{d \hat{u}}{d r}+\frac{\hat{u}}{r}\right)+a \hat{c} .
\end{aligned}
$$

Integrating the equilibrium equation $\operatorname{div} \hat{\boldsymbol{\sigma}}=0$ and using the boundary condition $\hat{\sigma}_{r}(R)=0$ leads to the relation

$$
2 G \frac{\hat{u}(R)}{R}=\frac{3 K+4 G}{3}\left(\frac{d \hat{u}}{d r}+\frac{\hat{u}}{r}\right)+a \hat{c}(r) .
$$

Using the constitutive relation $(6.2)$ as well as the relation $\hat{\boldsymbol{j}}=-(D / k)(d \hat{\mu} / d r) \boldsymbol{e}_{r}$, the diffusion equation gives

$$
\frac{k}{D a}\left(i \omega \hat{c}-\frac{D}{r} \frac{d}{d r}\left(r \frac{d \hat{c}}{d r}\right)\right)=\frac{d^{2}}{d r^{2}}\left(\frac{d \hat{u}}{d r}+\frac{\hat{u}}{r}\right)+\frac{1}{r} \frac{d}{d r}\left(\frac{d \hat{u}}{d r}+\frac{\hat{u}}{r}\right) .
$$

Eliminating $\frac{d \hat{u}}{d r}+\frac{\hat{u}}{r}$ between (6.7) and (6.8) leads to the Bessel differential equation

$$
\frac{i \omega}{D\left(1-\frac{3 a^{2}}{k(3 K+4 G)}\right)} r \hat{c}=\frac{d \hat{c}}{d r}+r \frac{d^{2} \hat{c}}{d r^{2}} .
$$

Bounded solutions to Eq.(6.9) take the form

$$
\hat{c}(r)=A J_{0}(\hat{\lambda} r)
$$


where $J$ is the Bessel function of the first kind and

$$
\hat{\lambda}=e^{i \frac{3 \pi}{4}} \sqrt{\frac{\omega}{D(1-a \tilde{a} / k)}}, \quad \tilde{a}=\frac{3 a}{3 K+4 G} .
$$

Substituting (6.10) in (6.7) allows the displacement $\hat{u}$ to be obtained. The constant $A$ in $(6.10)$ is obtained from the boundary condition $\boldsymbol{e}_{r} \cdot \hat{\boldsymbol{\jmath}}=i R \omega H / 4$ at $r=R$. The final expressions are

$$
\hat{c}=-\frac{H}{4} \frac{\hat{R} J_{0}(\hat{r})}{J_{1}(\hat{R})}, \quad \frac{\hat{u}}{R}=\tilde{a} \frac{H}{4}\left((1-2 \nu) \frac{\hat{r}}{\hat{R}}+\frac{J_{1}(\hat{r})}{J_{1}(\hat{R})}\right)
$$

where $\hat{r}=\hat{\lambda} r$ and $\hat{R}=\hat{\lambda} R$. Replacing in 6.6 gives the expression of the stress $\hat{\boldsymbol{\sigma}}$ as

$$
\begin{aligned}
& \hat{\sigma}_{r}=\frac{G \tilde{a} H}{2}\left(1-\frac{\hat{R}}{\hat{r}} \frac{J_{1}(\hat{r})}{J_{1}(\hat{R})}\right), \\
& \hat{\sigma}_{\theta}=\frac{G \tilde{a} H}{2}\left(1+\frac{\hat{R}}{\hat{r}} \frac{J_{1}(\hat{r})}{J_{1}(\hat{R})}-\frac{\hat{R} J_{0}(\hat{r})}{J_{1}(\hat{R})}\right), \\
& \hat{\sigma}_{z}=\frac{G \tilde{a} H}{2}\left(2 \nu-\frac{\hat{R} J_{0}(\hat{r})}{J_{1}(\hat{R})}\right) .
\end{aligned}
$$

The chemical potential $\hat{\mu}$ is obtained from the constitutive relations 6.2 as

$$
\hat{\mu}=\frac{H}{4}\left((a \tilde{a}-k) \frac{\hat{R} J_{0}(\hat{r})}{J_{1}(\hat{R})}+2(1-2 \nu) a \tilde{a}\right) .
$$

The obtained $T$-periodic response $\left(\boldsymbol{\sigma}^{E}, \mu^{E}, \boldsymbol{u}^{E}, c^{E}\right)$ is directly related to the large-time behavior in pure elasto-diffusion. As a particular case of the results of Sect. 4, the fields of stress and chemical potential in pure elasto-diffusion are indeed expected to converge to a cyclic steady state $\left(\boldsymbol{\sigma}_{\infty}, \mu_{\infty}\right)$ such that

$$
\boldsymbol{\sigma}_{\infty}(\boldsymbol{x}, t)=\boldsymbol{\sigma}^{E}(\boldsymbol{x}, t)+\boldsymbol{\rho}(\boldsymbol{x}), \quad \mu_{\infty}(\boldsymbol{x}, t)=\mu^{E}(\boldsymbol{x}, t)+\eta
$$

where $\boldsymbol{\rho}$ is a time-independent self-equilibrated stress field and $\eta$ is a constant. Using the constitutive equations shows that $\boldsymbol{\rho}$ and $\eta$ satisfy

$$
\boldsymbol{\rho}=\mathbb{L}: \boldsymbol{\varepsilon}\left(\left\langle\boldsymbol{u}_{\infty}\right\rangle\right)+a\left\langle c_{\infty}\right\rangle \boldsymbol{I}, \quad \eta=k\left\langle c_{\infty}\right\rangle+a \operatorname{tr} \varepsilon\left(\left\langle\boldsymbol{u}_{\infty}\right\rangle\right)
$$

where $\left\langle c_{\infty}\right\rangle$ (resp. $\left.\left\langle\boldsymbol{u}_{\infty}\right\rangle\right)$ denotes the time average of the lithium concentration $c_{\infty}$ (resp. displacement $\left.\boldsymbol{u}_{\infty}\right)$ in the cyclic steady state. The tensor $\boldsymbol{I}$ in 6.15 denotes the secondorder identity tensor. Let us find the values of $\boldsymbol{\rho}$ and $\eta$ corresponding to a lithium-free initial configuration. Reproducing the calculations used for finding $\hat{c}$ and $\hat{\boldsymbol{u}}$ shows that

$$
\boldsymbol{\rho}=(1-2 \nu) a\left\langle c_{\infty}\right\rangle \boldsymbol{e}_{z} \otimes \boldsymbol{e}_{z}, \quad \eta=(k-2(1-\nu) a \tilde{a})\left\langle c_{\infty}\right\rangle
$$


and

$$
\left\langle\boldsymbol{u}_{\infty}\right\rangle=-(1-\nu) \tilde{a}\left\langle c_{\infty}\right\rangle r \boldsymbol{e}_{r} .
$$

For a lithium-free initial state, the volume average of the lithium concentration is $\bar{c}(t)=$ $-\frac{2}{R} \int_{0}^{t} J\left(t^{\prime}\right) d t^{\prime}=\frac{1}{2} H(1-\cos \omega t)$. The time average of $\bar{c}(t)$ over a cycle is thus equal to $H / 2$. It follows that $c_{\infty}=H / 2$, which sets the value of the constant $\eta$ in (6.16). The cyclic steady state reached from a lithium-free initial state is thus given by

$$
\begin{aligned}
& \boldsymbol{\sigma}_{\infty}(r, t)=\frac{H}{2}(1-2 \nu) a \boldsymbol{e}_{z} \otimes \boldsymbol{e}_{z}+\Re\left(\hat{\boldsymbol{\sigma}}(r) e^{i \omega t}\right), \\
& \mu_{\infty}(r, t)=\mu_{0}+\frac{H}{2}(k-2(1-\nu) a \tilde{a})+\Re\left(\hat{\mu}(r) e^{i \omega t}\right), \\
& \boldsymbol{u}_{\infty}(r, t)=-\frac{H}{2}(1-\nu) \tilde{a} r \boldsymbol{e}_{r}+\Re\left(\hat{\boldsymbol{u}}(r) e^{i \omega t}\right), \\
& c_{\infty}(r, t)=\frac{H}{2}+\Re\left(\hat{c}(r) e^{i \omega t}\right) .
\end{aligned}
$$

The solution (6.17) complements existing analytical results for electrode particles in elasto-diffusion. Let us briefly study the influence of the charging rate $\omega$ on the obtained solution, focusing only on the time-dependent terms $\Re\left(\hat{c}(r) e^{i \omega t}\right)$ and $\Re\left(\hat{\boldsymbol{\sigma}}(r) e^{i \omega t}\right)$. In view of (6.11), it is natural to introduce the time scale

$$
T_{0}=\frac{R^{2}}{D\left(1-\frac{a \tilde{a}}{k}\right)} .
$$

In the following, the results are presented in terms of the dimensionless charging rate $\tilde{\omega}$ defined by $\tilde{\omega}=\omega T_{0}$.

The magnitude $|\hat{c}|$ is shown in Fig. 2 as a function of $r / R$ for several values of the dimensionless charging rate $\tilde{\omega}$. For low values of $\tilde{\omega}, \hat{c}$ is almost uniform and approximatively equal to $-H / 2$. This implies that $c_{\infty}(r, t) \simeq \frac{1}{2} H(1-\cos \omega t)$ : The insertion rate is so slow that diffusion equilibrium is reached at each time. As $\tilde{\omega}$ increases, $|\hat{c}|$ displays a monotonically increasing behavior with $r$, meaning that the time fluctuations of $c_{\infty}(r, t)$ get more pronounced as $r$ approaches the boundary. For high values of $\tilde{\omega}(>50),|\hat{c}|(r)$ is almost zero in the core of the cylinder, i.e. the lithium ions are concentrated in the outer shell of the particle. The asymptotic expression of $\hat{c}$ in the limit $\tilde{\omega} \rightarrow \infty$ can be calculated using a variation of the stationary phase method (Wong, 2001), yielding

$$
\hat{c}(r) \sim-\frac{H}{4} \sqrt{\frac{\tilde{\omega}}{\tilde{r}}} e^{i \frac{\pi}{4}+\frac{(1+i)}{\sqrt{2}} \sqrt{\tilde{\omega}}(\tilde{r}-1)}
$$

where $\tilde{r}=r / R$. The expression 6 (6.19) shows that the concentration of $|\hat{c}|$ at the boundary grows as $\sqrt{\tilde{\omega}}$.

In Fig. 3 are shown the magnitude $\left|\hat{\sigma}_{\theta}\right|$ and the relative phase $\Phi_{\theta}$ between the hoop stress $\sigma_{\theta}$ and the loading $J(t)$. The magnitude $\left|\hat{\sigma}_{\theta}\right|$ is not monotonically increasing with $r$ 
but still reaches its maximum at $r=R$. For low values of $\tilde{\omega}, \hat{\sigma}_{\theta}$ can be approximated by the first order expansion of 6.13 as

$$
\hat{\sigma}_{\theta} \sim i \frac{G \tilde{a} H}{16} \tilde{\omega}\left(1-3 \tilde{r}^{2}\right)
$$

The expression 6.20 shows that the hoop stress in the inner shell $r<R \sqrt{3}$ is in antiphase with the hoop stress in the outer shell $r>R \sqrt{3}$ : The inner shell is in circumferential traction (resp. compression) when the outer shell is in circumferential compression (resp. traction). If $a<0$ (i.e. lithiation results in volumetric expansion), the expression (6.20) also shows that the hoop stress at the boundary is in phase with the prescribed flux $J(t)$ : the outer surface $r=R$ is in circumferential compression (resp. traction) when lithiation (resp. delithiation) occurs.

For high values of $\tilde{\omega}$, we obtain

$$
\hat{\sigma}_{\theta} \sim \frac{G \tilde{a} H}{2}\left(1-\sqrt{\frac{\tilde{\omega}}{\tilde{r}}} e^{i \frac{\pi}{4}+\frac{(1+i)}{\sqrt{2}} \sqrt{\tilde{\omega}}(\tilde{r}-1)}\right) .
$$

The expression (6.21) shows that $\left|\hat{\sigma}_{\theta}\right|(r)$ is approximatively equal to $G|\tilde{a}| H / 2$ except in the outer shell where the hoop stress concentrates. On the outer surface $r=R$, Eq. (6.21) yields $\left|\hat{\sigma}_{\theta}(R)\right| \sim \sqrt{\tilde{\omega}} G|\tilde{a}| H / 2$ as $\tilde{\omega} \rightarrow \infty$, hence the hoop stress concentration grows as $\sqrt{\tilde{\omega}}$. It can also be observed in Fig. 3 (right) that the phase shift $\Phi_{\theta}(R, t)-\Phi_{\theta}(0, t)$ remains relatively close to $\pi$, whatever $\tilde{\omega}$ is: The hoop stress in the center is almost in antiphase with the hoop stress on the boundary, even for high values of $\tilde{\omega}$.

The stress $\hat{\sigma}_{z}$ is shown in Fig. 4 in the case $\nu=0.3$. For low values of $\tilde{\omega}$, the expression 6.13) gives

$$
\hat{\sigma}_{z} \sim i \frac{G \tilde{a} H}{2}\left(\frac{\tilde{\omega}}{4}\left(1-2 \tilde{r}^{2}\right)+2 i(1-\nu)\right) .
$$

For high values of $\tilde{\omega}$, we obtain

$$
\hat{\sigma}_{z} \sim \frac{G \tilde{a} H}{2}\left(2 \nu-\sqrt{\frac{\tilde{\omega}}{\tilde{r}}} e^{i \frac{\pi}{4}+\frac{(1+i)}{\sqrt{2}} \sqrt{\tilde{\omega}}(\tilde{r}-1)}\right) .
$$

In a way similar to the hoop stress, $\hat{\sigma}_{z}$ displays a concentration (growing as $\sqrt{\tilde{\omega}}$ ) at the boundary. Comparing Fig. 3 (right) with Fig. 4 (right) further shows that the phases $\Phi_{\theta}(r)$ and $\Phi_{z}(r)$ almost coincide for high values of $\tilde{\omega}$, consistently with 6.21 and 6.22.

Finally, the radial stress $\hat{\sigma}_{r}$ is shown in Fig. 5. For low values of $\tilde{\omega}$, we have

$$
\hat{\sigma}_{r} \sim i \frac{G \tilde{a} H}{16}\left(1-\tilde{r}^{2}\right)
$$

hence $\left|\hat{\sigma}_{r}\right|$ is maximum at the center. When $\tilde{\omega}$ increases, the value of $r$ maximizing $\left|\hat{\sigma}_{r}\right|$ moves towards the boundary. For high values of $\tilde{\omega}$, we obtain

$$
\hat{\sigma}_{r} \sim \frac{G \tilde{a} H}{2}\left(1-e^{\frac{(1+i)}{\sqrt{2}} \sqrt{\tilde{\omega}}(\tilde{r}-1)} \tilde{r}^{-\frac{3}{2}}\right)
$$


Thus, in contrast with $\hat{\sigma}_{\theta}$ and $\hat{\sigma}_{z}$, the magnitude of $\hat{\sigma}_{r}$ remains bounded as $\tilde{\omega} \rightarrow \infty$. It can also be observed in Fig. 5 (right) that the fluctuations of the phase $\Phi_{r}$ with $r$ are much smaller than for the other stress components.

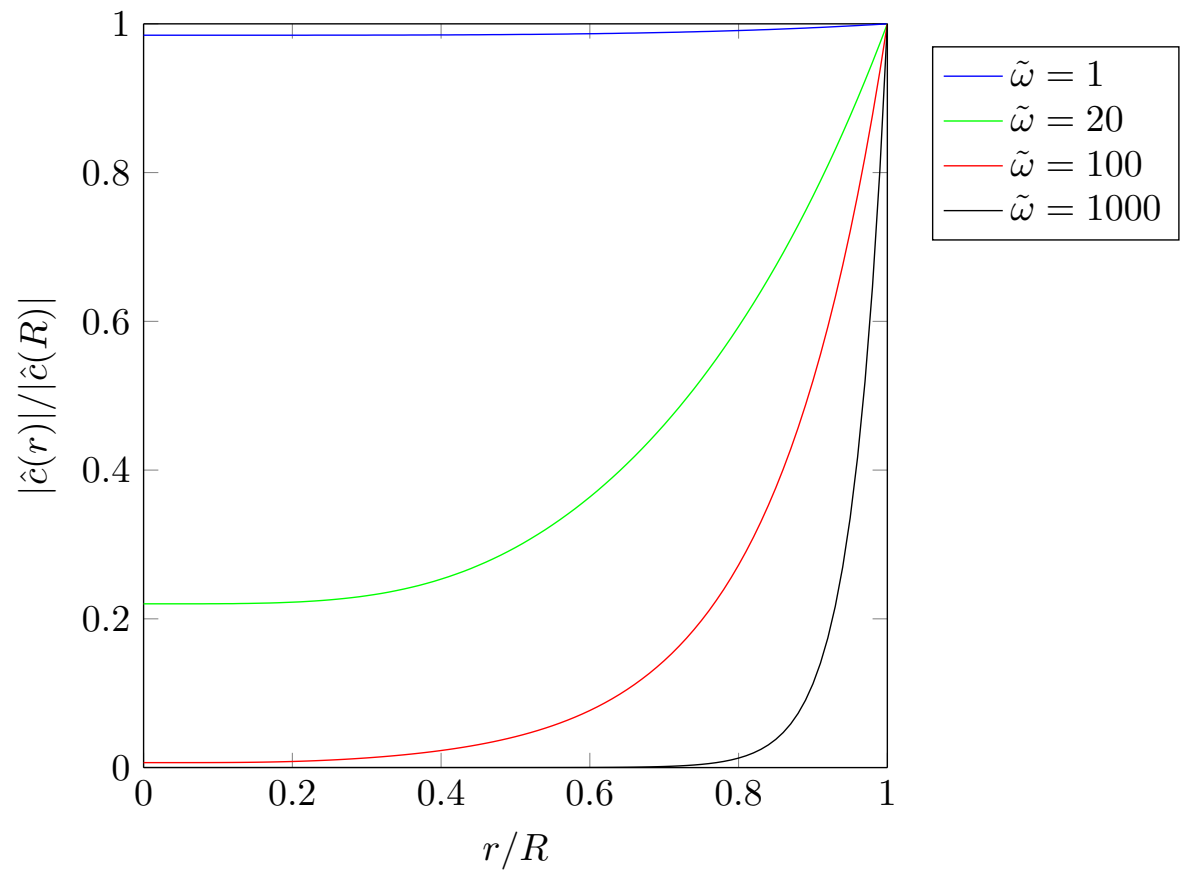

Figure 2: Magnitude of the complex concentration $\hat{c}$ of lithium as a function of $r / R$, for several values of the dimensionless charging rate $\tilde{\omega}$. The quantity $|\hat{c}| / H$ measures the time-fluctuations of the local concentration $c$ of lithium in the cyclic steady-state.

\subsubsection{Application of the shakedown condition}

Let now consider the case of an elastic-plastic behavior. We detail the application of the shakedown conditions introduced in Sect. 5.1 for an elasticity domain $\mathcal{C}$ of the Von Mises type, i.e. defined by

$$
\frac{1}{2} s: s \leq \sigma_{Y}^{2}
$$

where $\sigma_{Y}$ is the yield strength. All the considerations presented in this section are not restricted to harmonic loadings and apply for any chemo-mechanical loading that respects the circular symmetry. In such case, a $T$-periodic stress response to the purely elastodiffusive problem can be obtained in the form

$$
\boldsymbol{\sigma}^{E}(\boldsymbol{x}, t)=\sigma_{r}^{E}(r, t) \boldsymbol{e}_{r} \otimes \boldsymbol{e}_{r}+\sigma_{\theta}^{E}(r, t) \boldsymbol{e}_{\theta} \otimes \boldsymbol{e}_{\theta}+\sigma_{z}^{E}(r, t) \boldsymbol{e}_{z} \otimes \boldsymbol{e}_{z}
$$



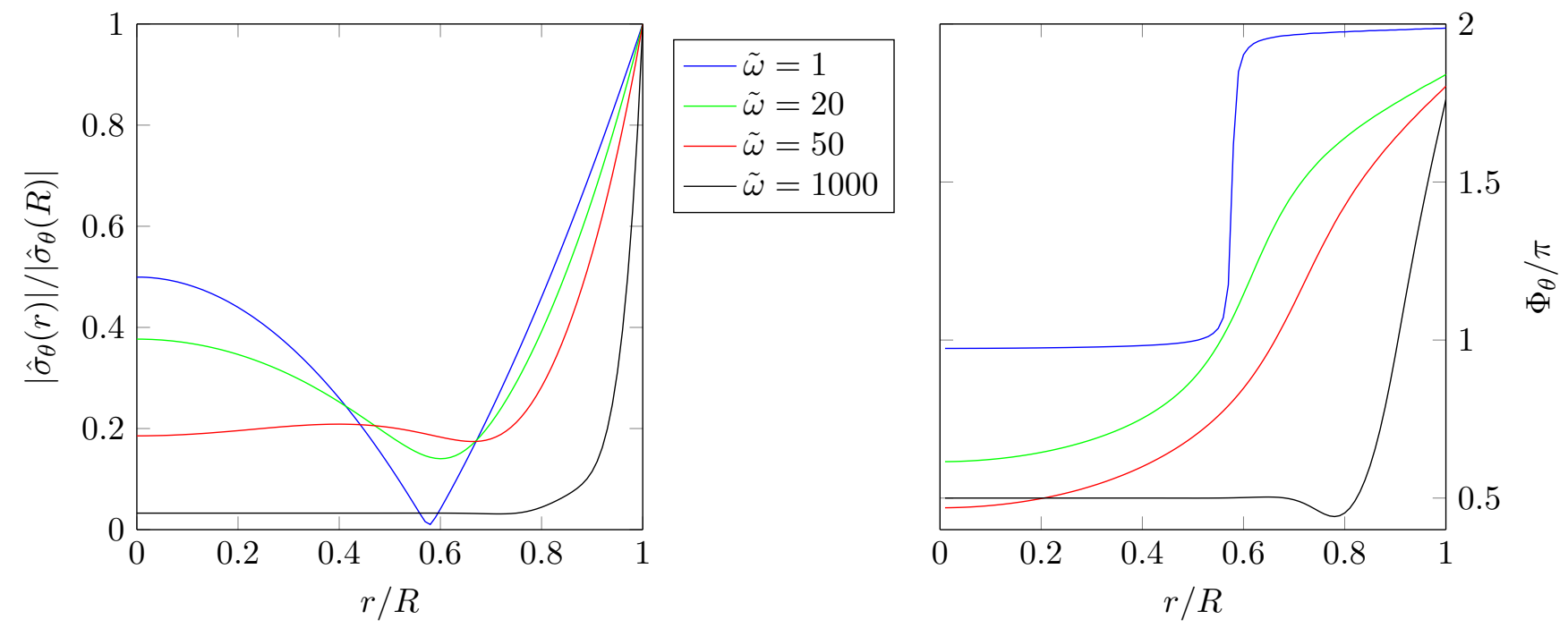

Figure 3: Magnitude (left) and phase (right) of the complex-valued hoop stress $\hat{\sigma}_{\theta}$ as a function of $r / R$, for several values of the dimensionless charging rate $\tilde{\omega}$.

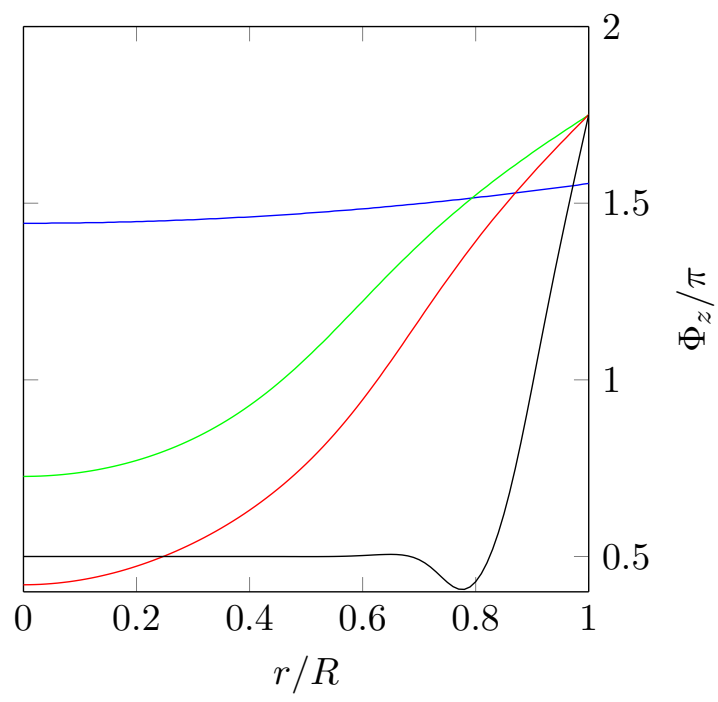

Figure 4: Magnitude (left) and phase (right) of the complex-valued stress $\hat{\sigma}_{z}$ as a function of $r / R$, for several values of the dimensionless charging rate $\tilde{\omega}$.

The corresponding deviatoric stress $\boldsymbol{s}^{E}$ is parameterized by its two components $\left(s_{r}^{E}, s_{\theta}^{E}\right)$ as

$$
\boldsymbol{s}^{E}=s_{r}^{E} \boldsymbol{e}_{r} \otimes \boldsymbol{e}_{r}+s_{\theta}^{E} \boldsymbol{e}_{\theta} \otimes \boldsymbol{e}_{\theta}-\left(s_{r}^{E}+s_{\theta}^{E}\right) \boldsymbol{e}_{z} \otimes \boldsymbol{e}_{z}
$$

where the dependence on $(x, t)$ has been omitted in the notations. 

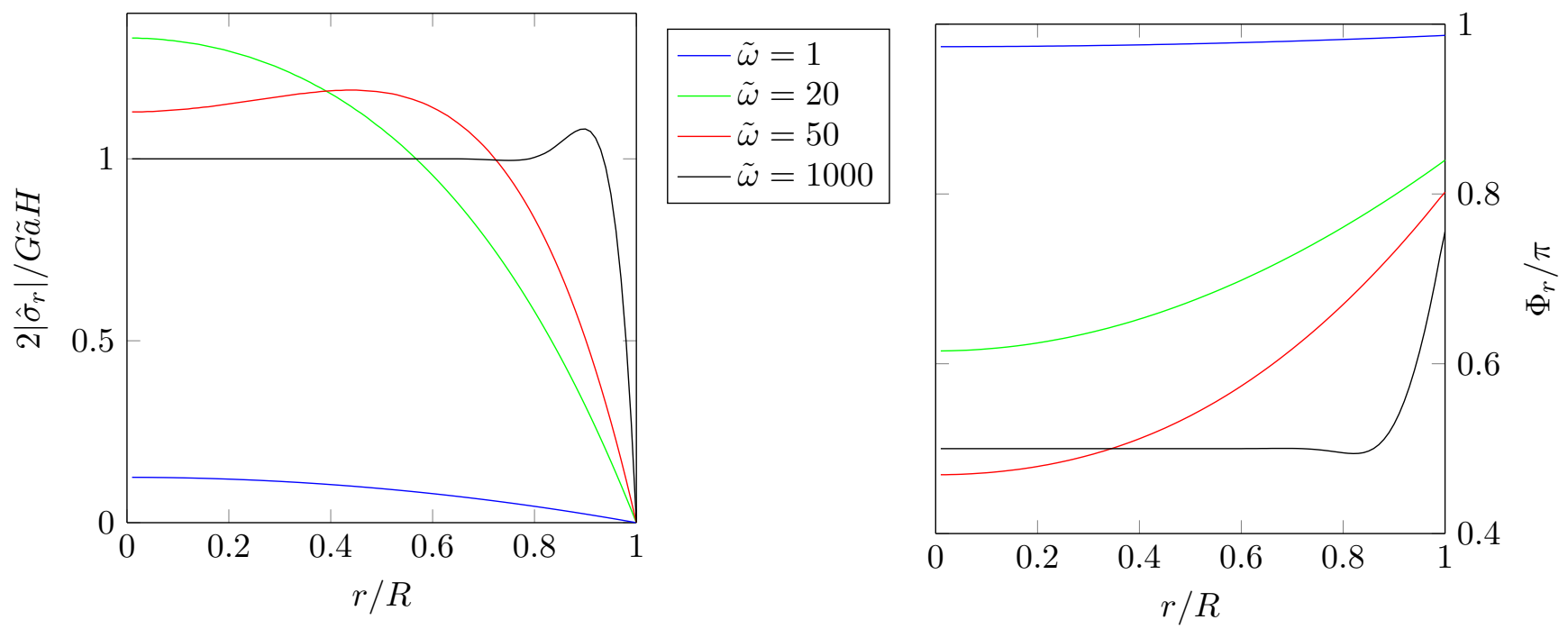

Figure 5: Magnitude (left) and phase (right) of the complex-valued radial stress $\hat{\sigma}_{r}$ as a function of $r / R$, for several values of the dimensionless charging rate $\tilde{\omega}$.

To fix ideas, we consider a rate-independent plastic behavior. Following the observations made in Sect. 5.3, checking the relevant shakedown condition 2 amounts to finding a timeindependent self-equilibrated stress field $\rho$ such that

$$
\left(\rho_{r}^{d}+s_{r}^{E}\right)^{2}+\left(\rho_{\theta}^{d}+s_{\theta}^{E}\right)^{2}+\left(\rho_{r}^{d}+\rho_{\theta}^{d}+s_{r}^{E}+s_{\theta}^{E}\right)^{2}+2\left(\rho_{r \theta}^{2}+\rho_{r z}^{2}+\rho_{\theta z}^{2}\right)<2 \sigma_{Y}^{2}
$$

for all $\boldsymbol{x}$ and all $t \in[0, T]$. In (6.24), $\boldsymbol{\rho}^{d}$ denotes the deviatoric part of $\boldsymbol{\rho}$. Eq. (6.24) implies that

$$
\left(\rho_{r}^{d}+s_{r}^{E}\right)^{2}+\left(\rho_{\theta}^{d}+s_{\theta}^{E}\right)^{2}+\left(\rho_{r}^{d}+\rho_{\theta}^{d}+s_{r}^{E}+s_{\theta}^{E}\right)^{2}<2 \sigma_{Y}^{2} .
$$

Setting

$$
\begin{array}{ll}
X^{E}=\sqrt{3}\left(s_{r}^{E}+s_{\theta}^{E}\right), & Y^{E}=s_{r}^{E}-s_{\theta}^{E}, \\
X^{0}=\sqrt{3}\left(\rho_{r}^{d}+\rho_{\theta}^{d}\right), & Y^{0}=\rho_{r}^{d}-\rho_{\theta}^{d}
\end{array}
$$

Eq. 6.25 can be rewritten as

$$
\left(X^{E}(r, t)+X^{0}(\boldsymbol{x})\right)^{2}+\left(Y^{E}(r, t)+Y^{0}(\boldsymbol{x})\right)^{2}<4 \sigma_{Y}^{2}
$$

for all $\boldsymbol{x}$ and all $t \in[0, T]$. Eq. (6.26) can be interpreted in geometrical terms: At each point $\boldsymbol{x}$, the local elastic stress path $t \mapsto\left(X^{E}, Y^{E}\right)(r, t)$ is enclosed by the disk with radius $2 \sigma_{Y}$ and centered at $\left(X^{0}(\boldsymbol{x}), Y^{0}(\boldsymbol{x})\right)$. Thus, denoting by $\gamma(r, \tilde{\omega})$ the radius of the smallest disk enclosing the curve $t \mapsto\left(X^{E}, Y^{E}\right)(r, t)$, we have

$$
\gamma(r, \tilde{\omega})<2 \sigma_{Y} \forall r \in[0, R) .
$$


The reasoning so far shows that the fulfillment of the shakedown condition 2 implies 6.27). The converse is also true: If (6.27) is satisfied for all $r \in[0, R)$, then there exists some time-independent functions $\left(\alpha_{r}(r), \alpha_{\theta}(r)\right)$ such that

$$
\left(\alpha_{r}+s_{r}^{E}\right)^{2}+\left(\alpha_{\theta}+s_{\theta}^{E}\right)^{2}+\left(\alpha_{r}+\alpha_{\theta}+s_{r}^{E}+s_{\theta}^{E}\right)^{2}<2 \sigma_{Y}^{2}
$$

for all $r \in[0, R)$ and $t \in[0, T]$. We claim that

$$
\alpha_{r}(r)=\rho_{r}^{d}(r), \alpha_{\theta}(r)=\rho_{\theta}^{d}(r)
$$

for some self-equilibrated stress field $\rho$. It can indeed be easily verified that the stress field $\rho$ defined by

$$
\boldsymbol{\rho}(r)=\left(\alpha_{\theta}(r)-\alpha_{r}(r)\right) \boldsymbol{e}_{\theta} \otimes \boldsymbol{e}_{\theta}-\left(\alpha_{\theta}(r)+2 \alpha_{r}(r)\right) \boldsymbol{e}_{z} \otimes \boldsymbol{e}_{z}-\left(\int_{r}^{R} \frac{\alpha_{\theta}(s)-\alpha_{r}(s)}{s} d s\right) \boldsymbol{I}
$$

fulfills 6.29 as well as the equilibrium equations $\operatorname{div} \boldsymbol{\rho}=0$ and $\rho_{r}(R)=0$. Consequently, the stress field $\rho$ in 6.30 is self-equilibrated and verifies (6.24), i.e. the shakedown condition 2 is satisfied.

Eq. 6.27) offers a practical shakedown criterion for the problem at hand. Let us now apply that criterion to the harmonic loading in (6.4), in which case a $T$-periodic stress response $\boldsymbol{\sigma}^{E}$ to the pure elasto-diffusion problem is given by Eqs. (6.5) and 6.13). In the following, the curves $t \mapsto\left(X^{E}, Y^{E}\right)(r, t)$ are referred to as stress loops and are denoted by $\Gamma(r, \tilde{\omega})$. Some stress loops are represented in Fig. 6 for several values of $r$ and $\tilde{\omega}$, in the case $\nu=0.3$. Two main observations are in order. The first one is that any given stress loop is symmetrical with respect to the origin $O$. This actually follows from the $T / 2$-antiperiodicity of the stress response $\boldsymbol{\sigma}^{E}(r, t)$. As a consequence of that symmetry, the smallest disk $\mathcal{D}$ enclosing a given stress loop $\Gamma(r, \tilde{\omega})$ is necessarily centered ${ }^{3}$ at $O$ so that its radius $\gamma(r, \tilde{\omega})$ is simply given by

$$
\gamma(r, \tilde{\omega})=\sup _{0 \leq t \leq T} \sqrt{\left(X^{E}(r, t)\right)^{2}+\left(Y^{E}(r, t)\right)^{2}}
$$

A second observation is that, at any given $\tilde{\omega}$, all the stress loops $\Gamma(r, \tilde{\omega})$ are enclosed in the stress loop $\Gamma(R, \tilde{\omega})$ (shown in red in Fig. 6). As a consequence, the condition (6.27) reduces to

$$
\gamma(R, \tilde{\omega})<2 \sigma_{Y}
$$

Using 6.31 and the expressions 6.13) of $\boldsymbol{\sigma}^{E}$, it can be calculated that

$$
\frac{\gamma(R, \tilde{\omega})}{G|\tilde{a}| H}=\frac{1}{\sqrt{6}}\left(A(\tilde{\omega})+\sqrt{A^{2}(\tilde{\omega})-B(\tilde{\omega})}\right)^{\frac{1}{2}}
$$

\footnotetext{
${ }^{3}$ Denoting the reflection through the origin by $S$, we have indeed $\Gamma(r, \tilde{\omega}) \subset \mathcal{D}$ hence $S \Gamma(r, \tilde{\omega}) \subset S \mathcal{D}$, i.e. $\Gamma(r, \tilde{\omega}) \subset S \mathcal{D}$. The disk $S \mathcal{D}$ has the same radius as $\mathcal{D}$. The uniqueness of the smallest disk enclosing $\Gamma(r, \tilde{\omega})$ then implies that $S \mathcal{D}=D$, i.e. that $D$ is centered at the origin.
} 

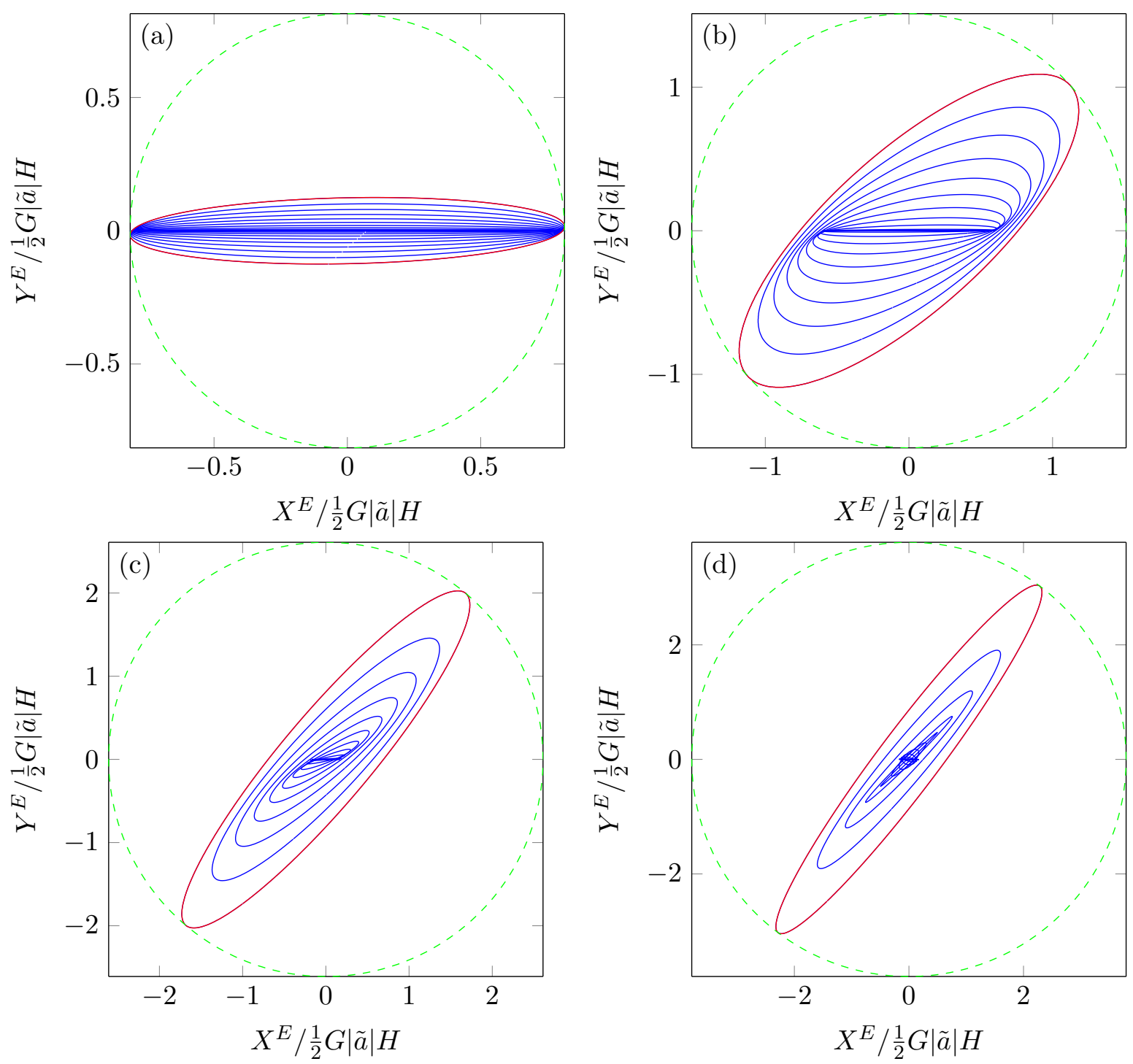

Figure 6: Stress loops $\Gamma(r, \tilde{\omega})$ for a harmonic loading with $\tilde{\omega}=1$ (a), $\tilde{\omega}=10$ (b), $\tilde{\omega}=25$ (c), $\tilde{\omega}=50$ (d). The blue curves show the stress loops at the location $r / R=i / 10$ with $i=0, \cdots 9$. The red curve is the stress loop at $r=R$. The smallest disk enclosing $\Gamma(R, \tilde{\omega})$ is shown as a dashed green curve.

where

$$
A(\tilde{\omega})=3(1-\nu)^{2}+(\Re z-1-\nu)^{2}+(\Im z)^{2}, B(\tilde{\omega})=12(1-\nu)^{2}(\Im z)^{2}
$$

and

$$
z=\frac{\hat{R} J_{0}(\hat{R})}{J_{1}(\hat{R})}
$$


From 6.32 , the shakedown domain in the $(\tilde{\omega}, H)$ map is defined by

$$
H<H_{S D}(\tilde{\omega})
$$

where

$$
H_{S D}(\tilde{\omega})=2 \frac{\sqrt{6} \sigma_{Y}}{G|\tilde{a}|}\left(A(\tilde{\omega})+\sqrt{A^{2}(\tilde{\omega})-B(\tilde{\omega})}\right)^{-\frac{1}{2}} .
$$

The shakedown limit $\tilde{\omega} \mapsto H_{S D}(\tilde{\omega})$ is shown as a solid line in Fig. 7. Even though rateindependent plasticity is considered, the loading rate $\tilde{\omega}$ has an influence on the shakedown limit. This unusual behavior results from the coupling with diffusion. It can also be observed that $H_{S D}(\tilde{\omega}) \rightarrow \infty$ as $\tilde{a} \rightarrow 0$. In that limiting case, the coupling between plasticity and diffusion vanishes so that a purely chemical loading does not induce any stress.

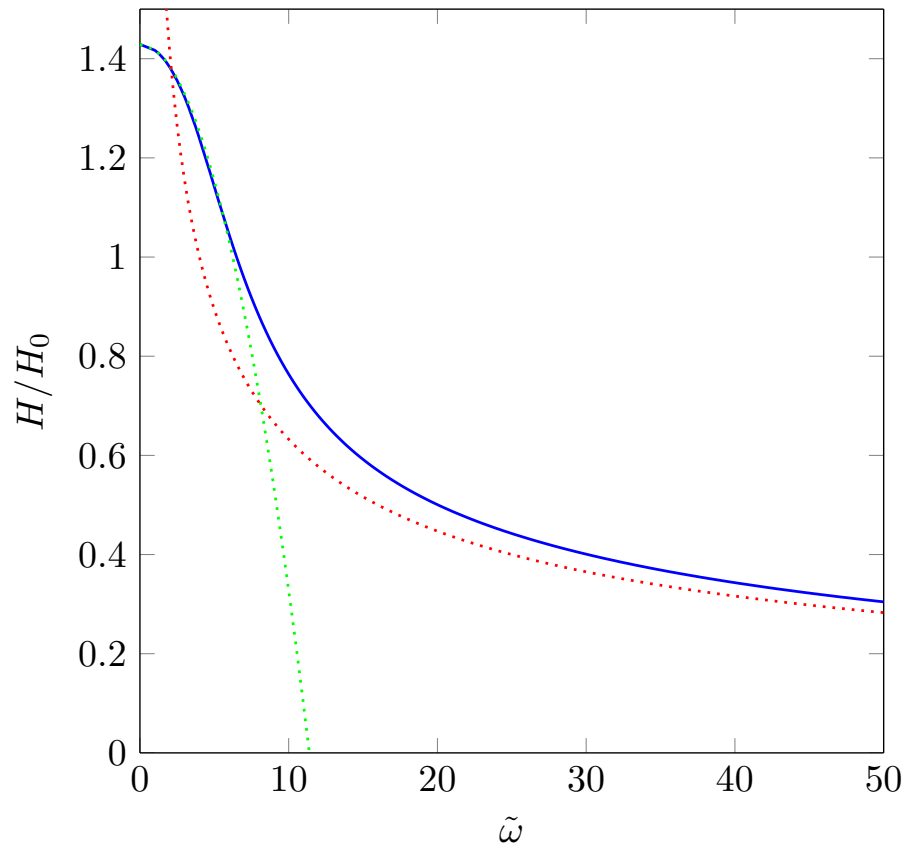

Figure 7: Shakedown domain for the harmonic loading, case $\nu=0.3$ : (solid line) shakedown limit $H_{S D}$; (dotted line) simplified expressions 6.376.38 in the limiting cases $\tilde{\omega} \rightarrow 0$ (green curve) and $\tilde{\omega} \rightarrow \infty$ (red curve).

The expression of the shakedown limit $H_{S D}(\tilde{\omega})$ can be simplified in the limiting cases $\tilde{\omega} \rightarrow 0$ and $\tilde{\omega} \rightarrow \infty$. For $\tilde{\omega} \rightarrow 0$, a Taylor expansion of $(6.36)$ gives

$$
\frac{H_{S D}(\tilde{\omega})}{H_{0}} \sim \frac{1}{1-\nu}-\frac{1}{2}\left(\frac{\tilde{\omega}}{8(1-\nu)}\right)^{2}\left(\frac{1}{4(1-\nu)}+\frac{1}{3}\right)
$$


where

$$
H_{0}=\frac{\sqrt{3} \sigma_{Y}}{G|\tilde{a}|}
$$

For $\tilde{\omega} \rightarrow \infty$, the asymptotic behavior of $H_{S D}(\tilde{\omega})$ is obtained from a variation of the stationary phase method (Wong, 2001) as

$$
\frac{H_{S D}(\tilde{\omega})}{H_{0}} \sim \frac{2}{\sqrt{\tilde{\omega}}}
$$

In Fig. 7, the shakedown limits provided by the simplified expressions (6.37) and (6.38) are shown as green and red dashed lines, respectively. To put things in perspective, consider a particle with a radius $R$ of $1 \mu \mathrm{m}$. For common electrodes materials such as $\mathrm{LiCoO}_{2}$ or $\mathrm{MnO}_{4}$, the diffusion parameter $D$ is of the order of $10^{-16} \mathrm{~m}^{2} / \mathrm{s}$ (Reimers and Dahn, 1992 Christensen and Newman, 2006). In the most favorable situation where $a \tilde{a} / k$ is negligible, the time scale $T_{0}$ in 6.18 is minimized and approximatively equal to $10^{4} \mathrm{~s}$. For batteries used in electronic devices, the typical charging/decharging period is in the range 1-10 h, which correspond to dimensionless charging rates $\tilde{\omega}$ in the range $2-20$. The maximum concentration of lithium in common electrodes materials is in the range $10^{4}-4.10^{4} \mathrm{~mol} / \mathrm{m}^{3}$. Using the representative values $E=80 \mathrm{GPa}, \nu=0.3, \sigma_{Y}=500 \mathrm{MPa}, a=-2.72 \times 10^{-18}$ $\mathrm{J}$, charging the cylinder particle at its maximum capacity then corresponds to values of $H$ in the range $5 H_{0}-20 H_{0}$. Such values are above the shakedown limit as can be observed in Fig. 7. For a charging time of $10 \mathrm{~h}(\tilde{\omega} \simeq 2)$ and a maximum concentration of $10^{4}$ $\mathrm{mol} / \mathrm{m}^{3}$, the particle can only be charged at approximatively $30 \%$ of its maximum capacity for remaining in the elastic shakedown regime. Those rather severe restrictions partly stem from the plane strain assumption used in the calculations and become less stringent for a stress free configuration, as will be further detailed in Sects. 6.3 6.4 below.

We emphasize that the fulfillment of the condition (6.35) ensures that shakedown occurs for any initial state, even if the latter does not respect the circular symmetry. To illustrate that point, finite-element calculations have been performed to obtain the evolution of the particle starting from two initial plastic strain fields, labeled as $\varepsilon_{A}^{p}$ and $\varepsilon_{B}^{p}$. The fields $\varepsilon_{A}^{p}$ and $\varepsilon_{B}^{p}$ are represented in Fig. 8. The exact expression of $\varepsilon_{A}^{p}$ is $\varepsilon_{A}^{p}=0.003 \sin (\pi r / 2 R)\left(\boldsymbol{e}_{1} \otimes\right.$ $\left.\boldsymbol{e}_{2}+\boldsymbol{e}_{2} \otimes \boldsymbol{e}_{1}\right)$. The field $\varepsilon_{B}^{p}$ consists in two circular inclusions with a uniform plastic strain $0.0006\left(\boldsymbol{e}_{1} \otimes \boldsymbol{e}_{2}+\boldsymbol{e}_{2} \otimes \boldsymbol{e}_{1}\right)$ in each inclusion. The material parameters used for the simulations are $E=80 \mathrm{GPa}, \nu=0.3, \sigma_{Y}=50 \mathrm{MPa}, a=-2.72 \times 10^{-18} \mathrm{~J}, k=0.5 \times 10^{-13} \mathrm{~J}^{3} \mathrm{~m}^{3}$, $D=10^{-16} \mathrm{~m}^{2} / \mathrm{s}$. The charging rate $\tilde{\omega}$ is set to $\tilde{\omega}=10$, in which case the shakedown limit predicted by Eq. (6.36) is approximatively equal to $0.75 H_{0}$. In Fig. 9 is plotted the evolution of the norm $\int_{\Omega}\left|\varepsilon^{p}(t)\right|$ of the plastic strain field, as estimated numerically for $H=0.7 H_{0}$, i.e. below the shakedown limit. As expected, the plastic strain field is found to reach a steady state value for both initial states, which is illustrated in Fig. 9. In Fig. 10 is plotted the evolution of the plastic strain rate field in the case $H=0.85 H_{0}$, i.e. above the shakedown limit. As expected, the plastic strain does not stabilize to a time-independent 

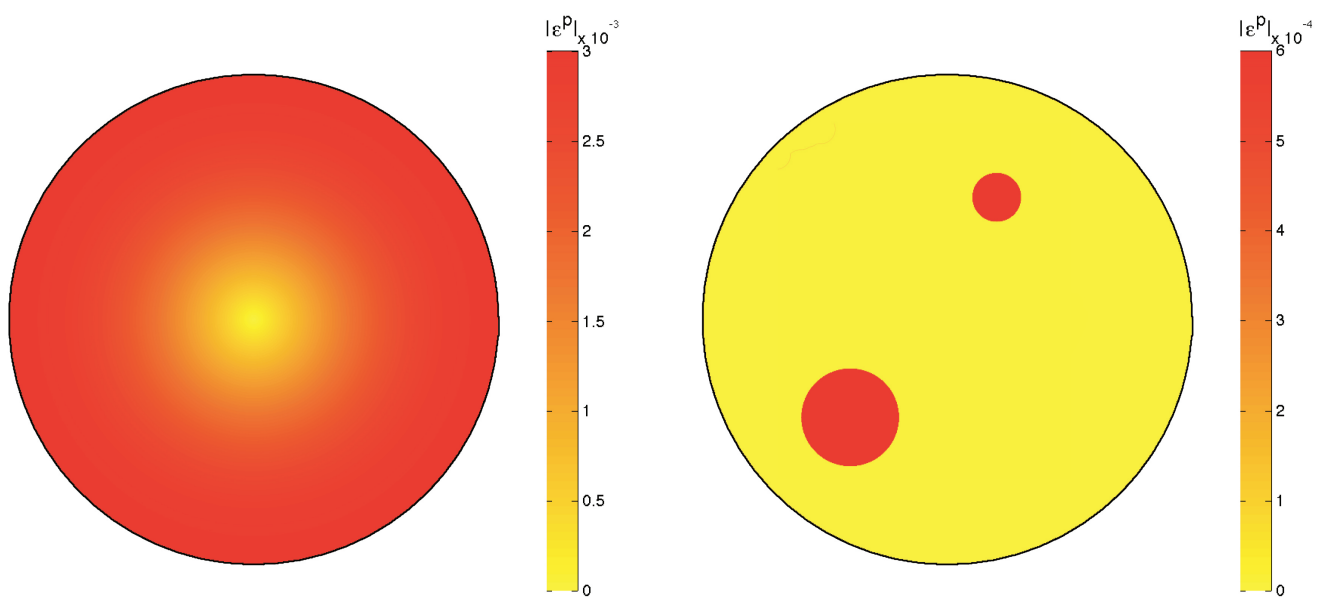

Figure 8: Initial plastic strain fields $\varepsilon_{A}^{p}$ and $\varepsilon_{B}^{p}$ used in the finite-element calculations.

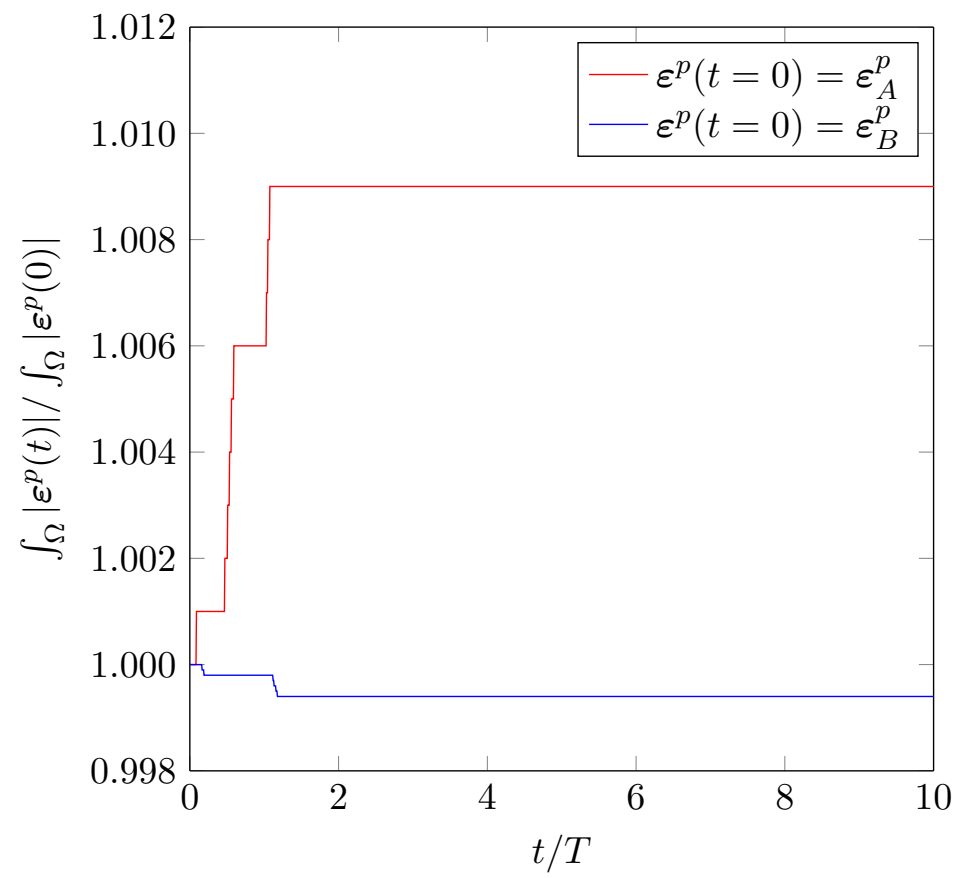

Figure 9: Time-evolution of the norm of the plastic strain field (normalized with respect to its value at $t=0$ ), for the two different initial states $\varepsilon_{A}^{p}$ and $\varepsilon_{B}^{p}$. The loading is harmonic with parameters $\tilde{\omega}=10$, $H=0.7 H_{0}$. 
value: As can be observed in Fig. 10, the plastic strain rate converges towards a non zero cyclic steady state that is the same for both initial states considered.

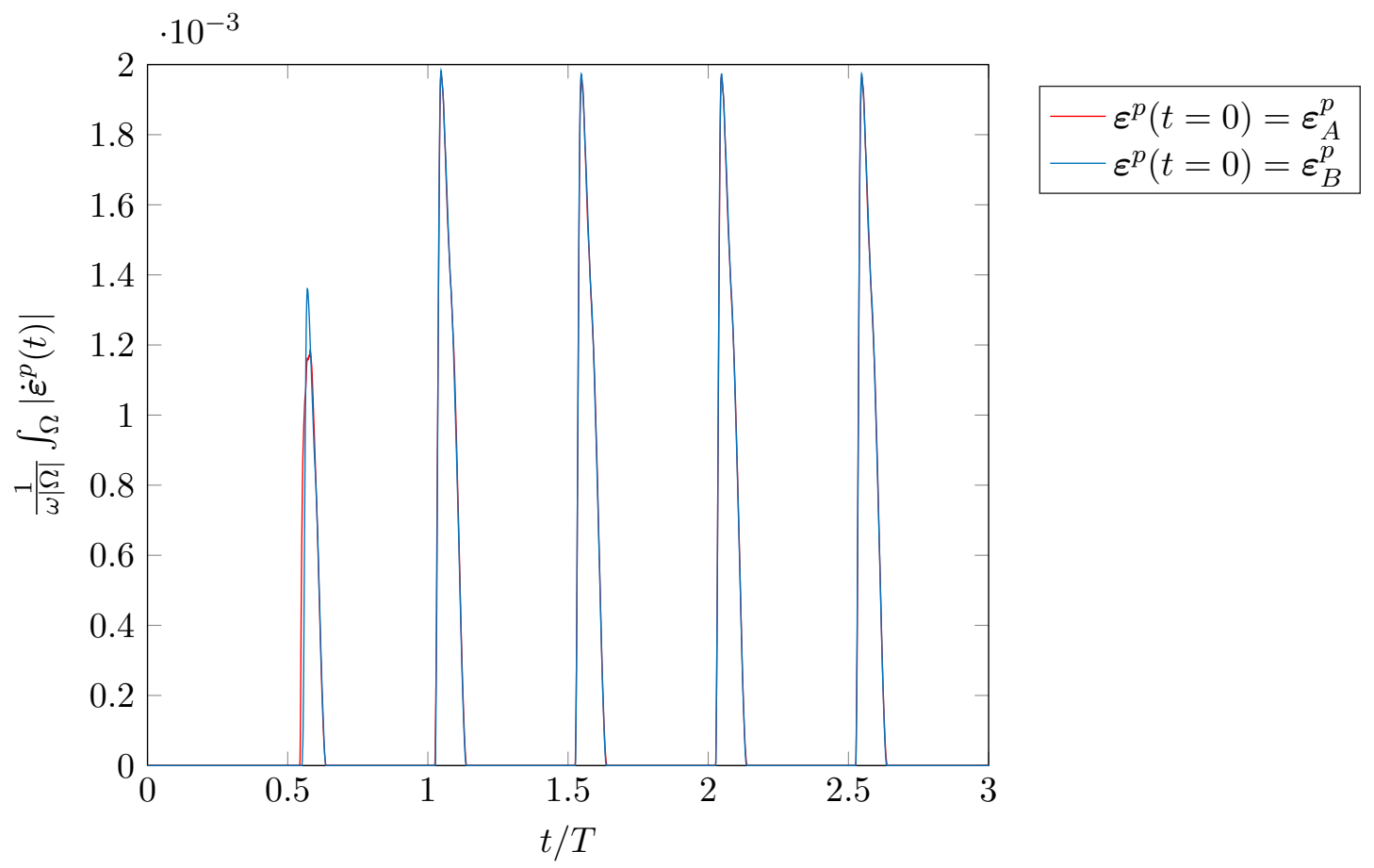

Figure 10: Time-evolution of the plastic strain rate field (in norm), for the two different initial states $\varepsilon_{A}^{p}$ and $\varepsilon_{B}^{p}$. The loading is harmonic with parameters $\tilde{\omega}=10, H=0.85 H_{0}$.

Remark: The presented analysis can be adapted to the case where the chemical potential $\mu$ is imposed at $r=R$, instead of the flux $J$. Writing the imposed chemical potential $\mu(R, t)$ as $\mu_{0}+M_{a} \sin \omega t$ where $M_{a}$ is the loading parameter, the shakedown limit $M_{S D}(\tilde{\omega})$ on $M_{a}$ is given by

$$
M_{S D}(\tilde{\omega})=\frac{H_{S D}(\tilde{\omega})}{4}((k-a \tilde{a})|z|+2(1-2 \nu) a \tilde{a})
$$

where $z=\hat{R} J_{0}(\hat{R}) / J_{1}(\hat{R})$. The shakedown limit $M_{S D}(\tilde{\omega})$ does not decrease monotonically with $\tilde{\omega}$. For instance, in the case $a \tilde{a} \ll k, M_{S D}(\tilde{\omega})$ increases for $\tilde{\omega}<\tilde{\omega}_{M}$ and decreases for $\tilde{\omega}>\tilde{\omega}_{M}$, with $\tilde{\omega}_{M} \simeq 3.1$. This non monotonic behavior comes from the fact that the terms $H_{S D}(\tilde{\omega})$ and $|z|$ that appear in $(6.39)$ vary in opposite fashion with $\tilde{\omega}$. In particular, $|z|$ is increasing with $\tilde{\omega}$, which means that the maximum number of lithium ions injected for a fixed value of $M_{a}$ is decreasing with $\tilde{\omega}$. 


\subsection{Smoothed square-wave loading}

As a further illustration, we use the solution of the harmonic problem to study the case of the smoothed square-wave loading $J(t)$ defined by

$$
J(t)=-\frac{R \omega H}{\tau} J_{0}(t)
$$

where

$$
J_{0}(t)=\sum_{n=1}^{N} \frac{1}{n}\left(\operatorname{sinc}^{2} \frac{n}{N}\right) \sin n \omega t
$$

and

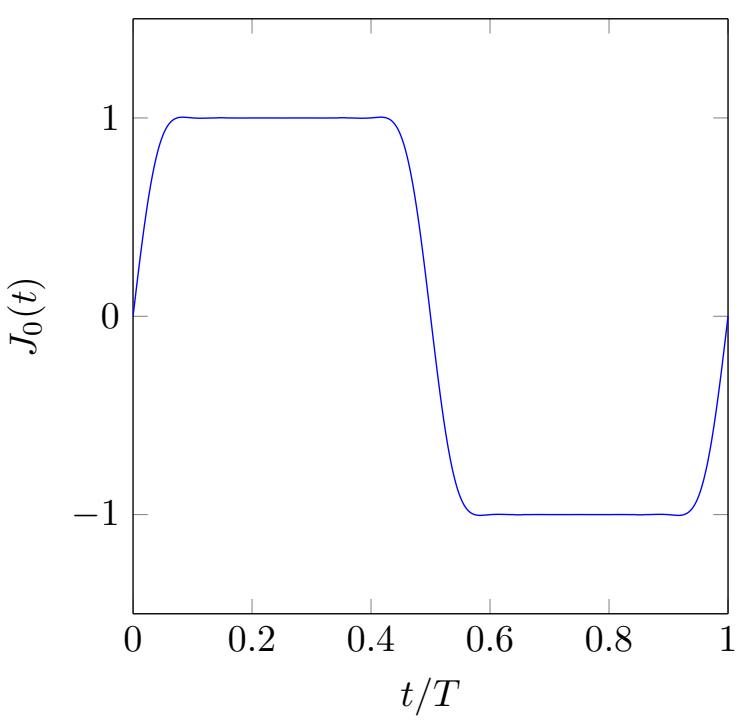

Figure 11: Smoothed square-wave loading.

$$
\tau=4 \sum_{\substack{n=1 \\ n \text { odd }}}^{N} \frac{1}{n^{2}}\left(\operatorname{sinc}^{2} \frac{n}{N}\right)
$$

In (6.41), $N$ is a fixed even integer. The function $J_{0}(t)$ in 6.41 is represented in Fig. 11 for $N=10$. In 6.40 , the dimensionless number $\tau$ is introduced in such fashion that the loading parameter $H$ is equal to the maximum number of inserted lithium ions (per unit volume). Compared to the harmonic case, the loading in Fig. 11 is more representative of situations where charge and discharge occur under a constant electric current. 
A $T$-periodic response $\left(\boldsymbol{\sigma}^{E}, \mu^{E}, \boldsymbol{u}^{E}, c^{E}\right)$ to the pure elasto-diffusion problem can be constructed using the procedure described in Sect. 5.2. For instance, the displacement $\boldsymbol{u}^{E}$ is given by

$$
\boldsymbol{u}^{E}=\sum_{\substack{n=1 \\ n \text { odd }}}^{N} \Re\left(\hat{u}_{n} e^{i n \omega t}\right) \boldsymbol{e}_{r}
$$

where $\hat{u}_{n}$ is obtained by replacing $(\tilde{\omega}, H)$ with $\left(n \tilde{\omega},\left(4 H / \tau n^{2}\right) \operatorname{sinc}^{2} \frac{n}{N}\right)$ in the expression of $\hat{u}$ in 6.12 . Similarly, the stress $\boldsymbol{\sigma}^{E}$ can be written as $\boldsymbol{\sigma}^{E}=\Re(\hat{\boldsymbol{\sigma}})$ where $\hat{\boldsymbol{\sigma}}=\hat{\sigma}_{r} \boldsymbol{e}_{r} \otimes \boldsymbol{e}_{r}+$ $\hat{\sigma}_{\theta} \boldsymbol{e}_{\theta} \otimes \boldsymbol{e}_{\theta}+\hat{\sigma}_{z} \boldsymbol{e}_{z} \otimes \boldsymbol{e}_{z}$ and

$$
\begin{aligned}
\frac{\hat{\sigma}_{r}}{G \tilde{a} H}= & \frac{2}{\tau} \sum_{\substack{n=1 \\
n \text { odd }}}^{N} \frac{1}{n^{2}}\left(\operatorname{sinc}^{2} \frac{n}{N}\right)\left(1-\frac{\hat{R}}{\hat{r}} \frac{J_{1}(\sqrt{n} \hat{r})}{J_{1}(\sqrt{n} \hat{R})}\right) e^{i n \omega t}, \\
\frac{\hat{\sigma}_{\theta}}{G \tilde{a} H}= & \frac{2}{\tau} \sum_{\substack{n=1 \\
n \text { odd }}}^{N} \frac{1}{n^{2}}\left(\operatorname{sinc}^{2} \frac{n}{N}\right)\left(1+\frac{\hat{R}}{\hat{r}} \frac{J_{1}(\sqrt{n} \hat{r})}{J_{1}(\sqrt{n} \hat{R})}-\sqrt{n} \hat{R} \frac{J_{0}(\sqrt{n} \hat{r})}{J_{1}(\sqrt{n} \hat{R})}\right) e^{i n \omega t}, \\
\frac{\hat{\sigma}_{z}}{G \tilde{a} H}= & \frac{2}{\tau} \sum_{\substack{n=1 \\
n \text { odd }}}^{N} \frac{1}{n^{2}}\left(\operatorname{sinc}^{2} \frac{n}{N}\right)\left(2 \nu-\frac{\sqrt{n} \hat{R} J_{0}(\sqrt{n} \hat{r})}{J_{1}(\sqrt{n} \hat{R})}\right) e^{i n \omega t} .
\end{aligned}
$$

Some stress loops corresponding to the expressions $\sqrt{6.42}$ are shown in Fig. 12 . Since the applied loading is $T / 2$-antiperiodic, every stress loop $\Gamma(r, \tilde{\omega})$ is symmetrical with respect to the origin $O$ so that the expression (6.31) of $\gamma(r, \tilde{\omega})$ still holds. As for the harmonic loading, we further observe that the stress loop $\Gamma(R, \tilde{\omega})$ encloses all the other stress loops $\Gamma(r, \tilde{\omega})$ at the same charging rate. The shakedown condition is thus

$$
\sup _{0 \leq t \leq T} \sqrt{\left(X^{E}(R, t)\right)^{2}+\left(Y^{E}(R, t)\right)^{2}}<\sqrt{2} \sigma_{Y}
$$

For any given $\tilde{\omega}$, the supremum in $(6.43)$ can be estimated numerically from the expressions 6.42). The resulting shakedown diagram is presented in Fig. 13(solid line) for the case $\nu=0.3$. When $\tilde{\omega}>7.5$, the smoothed square-wave loading is found to be more favorable than the harmonic loading, in the sense that the shakedown limit is higher. The conclusion is reversed when $\tilde{\omega}<7.5$. However, it should be observed that the relative difference between the shakedown limits for those two loadings remains small and never exceeds $6 \%$.

Consider some loading parameters $(\tilde{\omega}, H)$ that fall in the shakedown domain. In the large-time limit, the stress and chemical potential fields differ from the purely elastodiffusive response $\left(\boldsymbol{\sigma}^{E}, \mu^{E}\right)$ by some time-independent field $(\boldsymbol{\rho}, \eta) \in \mathcal{K}_{0}$. Up to a constant, 

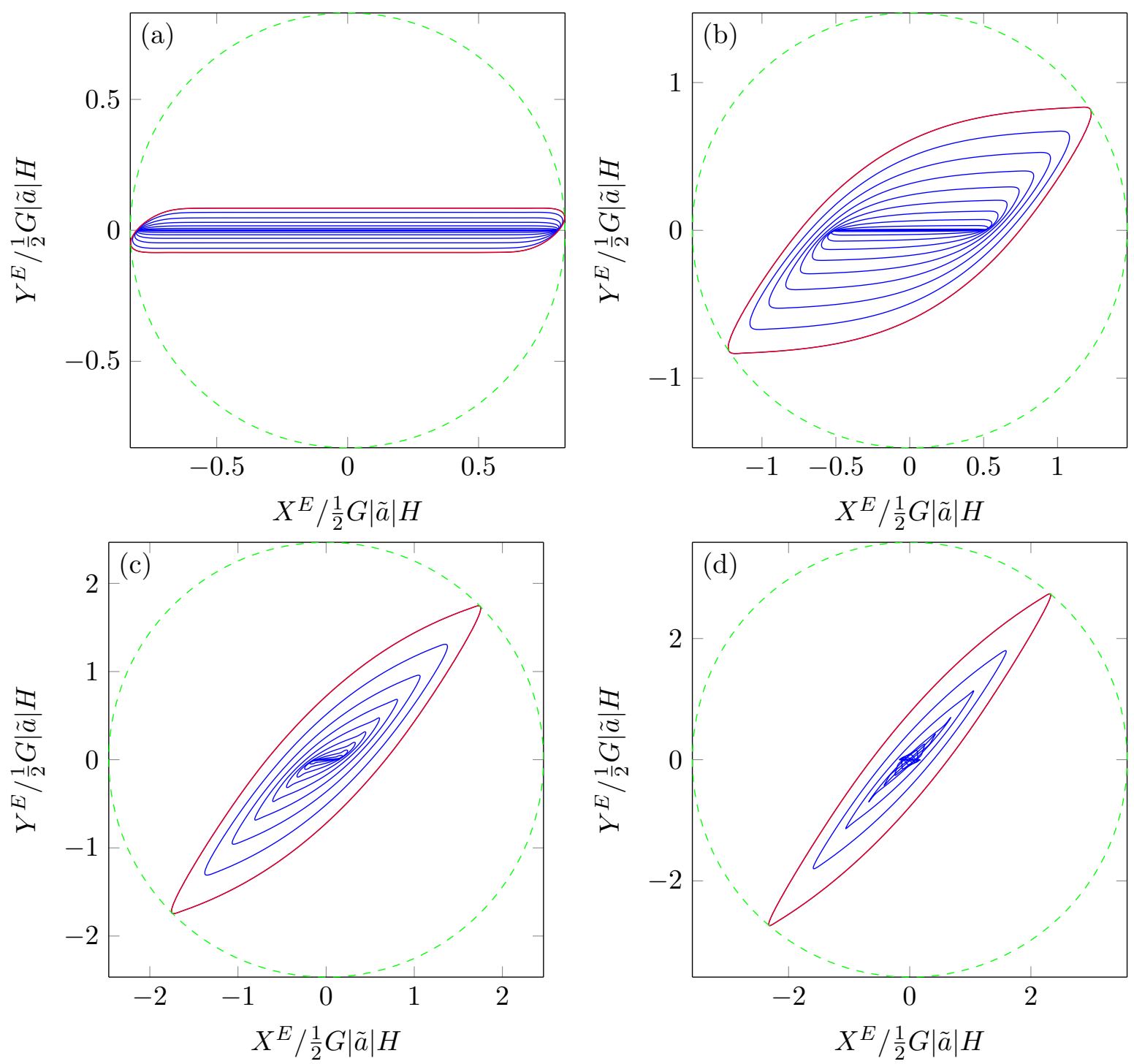

Figure 12: Stress loops $\Gamma(r, \tilde{\omega})$ for a smoothed square-wave loading with $\tilde{\omega}=1$ (a), $\tilde{\omega}=10$ (b), $\tilde{\omega}=25$ (c), $\tilde{\omega}=50(\mathrm{~d})$. The blue curves show the stress loops at the location $r / R=i / 10$ with $i=0, \cdots, 9$. The red curve is the stress loop at $r=R$. The smallest disk enclosing $\Gamma(R, \tilde{\omega})$ is shown as a dashed green curve.

the large-time expression of the chemical potential $\mu(R, t)$ on the surface $r=R$ is thus 


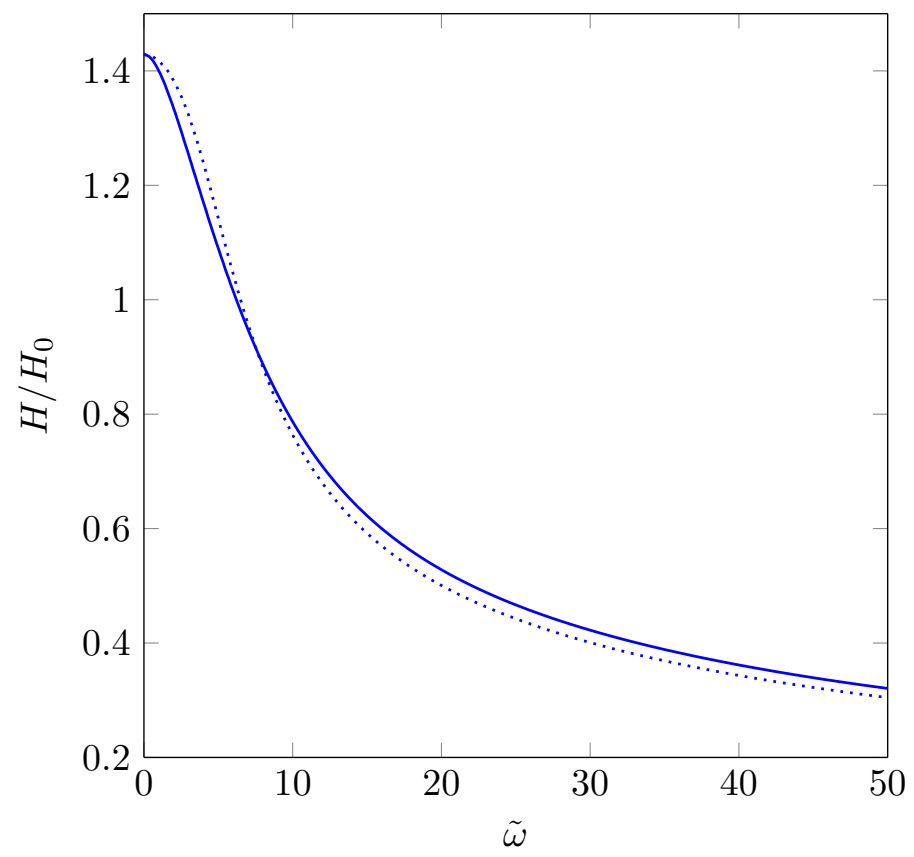

Figure 13: Shakedown map for a smoothed square-wave loading (solid line) and a harmonic loading (dotted line). Case $\nu=0.3$.

given by $\mu_{0}+\Re(\hat{\mu}(R, t))$ with

$$
\frac{\hat{\mu}(R, t)}{H}=\frac{1}{\tau} \sum_{\substack{n=1 \\ n \text { odd }}}^{N} \frac{1}{n^{2}}\left(\operatorname{sinc}^{2} \frac{n}{N}\right)\left((a \tilde{a}-k) \frac{\sqrt{n} \hat{R} J_{0}(\sqrt{n} \hat{R})}{J_{1}(\sqrt{n} \hat{R})}+2(1-2 \nu) a \tilde{a}\right) e^{i n \omega t} .
$$

The chemical potential $\mu(R, t)$ is directly related to the voltage $V(t)$ between the considered electrode particle and a reference electrode at a chemical potential $\mu_{0}$. We have indeed the relation

$$
V(t)=-\left(\mu(R, t)-\mu_{0}\right) / e
$$

where $e=1.602 \times 10^{-19} \mathrm{C}$ is the elementary charge. The voltage $V(t)$ corresponding to (6.44) is represented in Fig. 14 as a function of the average volume fraction $\bar{c}(t)$ of lithium, in the case $\tilde{a} a \ll k$. The curves in Fig. 14 can be interpreted as charge-voltage responses in the large-time limit. Changing the initial state may only result in a time-independent shift of the curves in the $(\bar{c}, V)$ plane. Those $\bar{c}-V$ curves hold provided that the loading parameters $(\tilde{\omega}, H)$ respect the shakedown condition $(6.43)$. In such a condition, the chargedischarge hysteresis that is observed in Fig, 14 results only from the diffusion and increases with the charging rate $\tilde{\omega}$. Compared with the curves obtained for harmonic loadings (shown 
as red dotted lines in Fig. 14), the $\bar{c}-V$ curves for the smoothed square-wave loading display a smaller hysteresis, which is especially visible for high values of $\tilde{\omega}$.

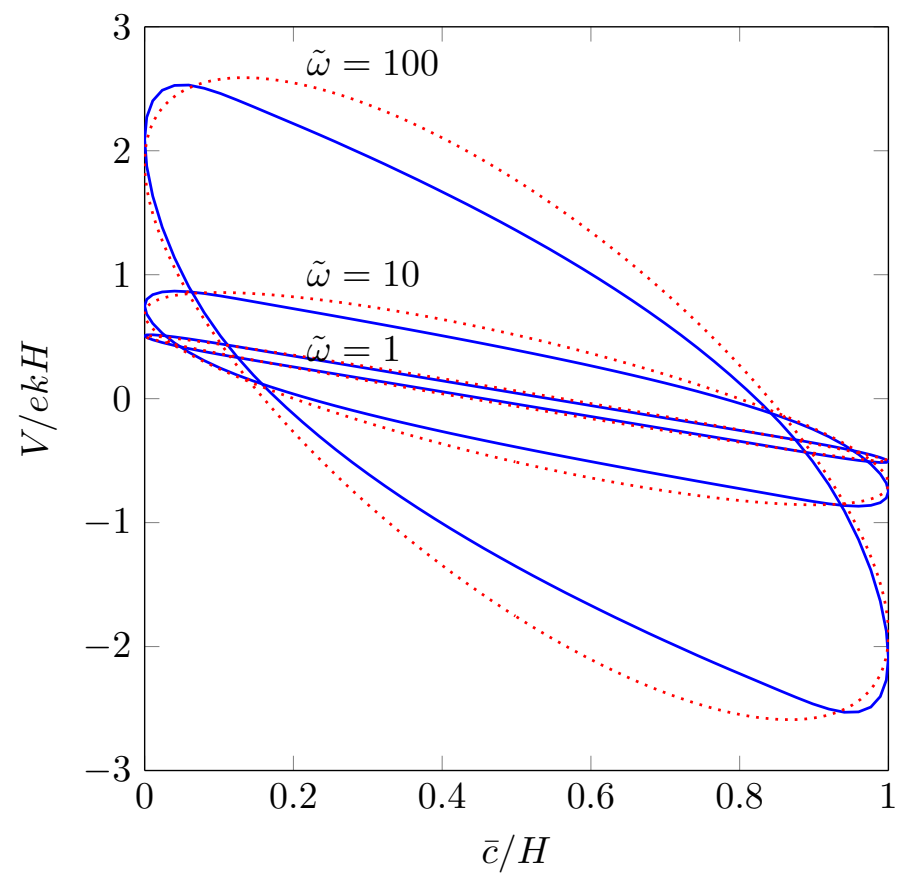

Figure 14: Charge-voltage response in the cyclic steady state of elastic shakedown, for a smoothed squarewave loading (solid line) and a harmonic loading (dotted line).

\subsection{Chemo-mechanical loading}

The loadings considered so far are purely chemical, but the shakedown condition 6.27) can be used in more general situations where both chemical and mechanical loadings are involved. To illustrate that point, we study an example of chemo-mechanical loading with two parameters. Specifically, the axial strain of the particle is assumed to vary cyclically in time due to prescribed normal tractions $\boldsymbol{T}(\boldsymbol{x}, t)$ applied on the top and bottom surfaces $(z=0, H)$. The normal force $\boldsymbol{F}(t)=\int_{z=H} \boldsymbol{T}(\boldsymbol{x}, t) d S$ on the top surface is written as $\boldsymbol{F}(t)=\pi R^{2} \sigma_{F} \cos \omega t \boldsymbol{e}_{z}$ when $\sigma_{F}$ is the mechanical loading parameter. Cyclic lithiation is imposed as in (6.4) where $H$ is the chemical loading parameter.

Omitting the detail of the calculations, the purely elasto-diffusive stress $\boldsymbol{\sigma}^{E}$ is found to be given by the same equations as (6.5) and (6.13) except that $\hat{\sigma}_{z}$ is now defined by

$$
\hat{\sigma}_{z}=\frac{G \tilde{a} H}{2}\left(2-\frac{\hat{R} J_{0}(\hat{r})}{J_{1}(\hat{R})}\right)+\sigma_{F} .
$$


Following the analysis reported in Sect. 6.1.2, shakedown occurs if $\gamma(r, \tilde{\omega})<2 \sigma_{Y}$ for all $r$. Assuming that $a<0$ (i.e. that lithiation results in volumetric expansion), $\gamma(r, \tilde{\omega})$ is found to be maximum at $r=R$ so that the shakedown condition reduces to $\gamma(R, \tilde{\omega})<2 \sigma_{Y}$. That last requirement is found to translate as

$$
\frac{1}{3}\left(\frac{\sigma_{F}}{\sigma_{Y}}\right)^{2}+\left(\frac{H}{H_{1}(\tilde{\omega})}\right)^{2}-\beta(\tilde{\omega}) \frac{\sigma_{F}}{\sigma_{Y}} \frac{H}{H_{1}(\tilde{\omega})}-\frac{1}{4}\left(1-3 \beta(\tilde{\omega})^{2}\right)\left(\frac{\sigma_{F}}{\sigma_{Y}} \frac{H}{H_{1}(\tilde{\omega})}\right)^{2}<1
$$

where

$$
H_{1}(\tilde{\omega})=\frac{2}{G|\tilde{a}|} \frac{\sqrt{3} \sigma_{Y}}{\sqrt{(\Re z-2)^{2}+(\Im z)^{2}}}, \beta(\tilde{\omega})=\frac{\sqrt{3}(\Re z-2)}{3 \sqrt{(\Re z-2)^{2}+(\Im z)^{2}}}
$$

and $z=\hat{R} J_{0}(\hat{R}) / J_{1}(\hat{R})$. Eq. 6.46 defines the shakedown domain in the space of loading parameters $\left(H, \sigma_{F}\right)$. The boundary of the shakedown domain is represented in Fig. 15 for several values of the loading rate $\tilde{\omega}$. For $H=0$, the shakedown limit on $\sigma_{F}$ is equal to $\sqrt{3} \sigma_{Y}$. For $\sigma_{F}=0$ (case of a free cylinder), the shakedown limit on the chemical loading parameter $H$ is equal to $H_{1}(\tilde{\omega})$ and becomes infinite when $\tilde{\omega}$ tends towards 0 . For later reference, the shakedown limit for the free cylinder is shown in Fig. 16 in the $(\tilde{\omega}, H)$ plane.

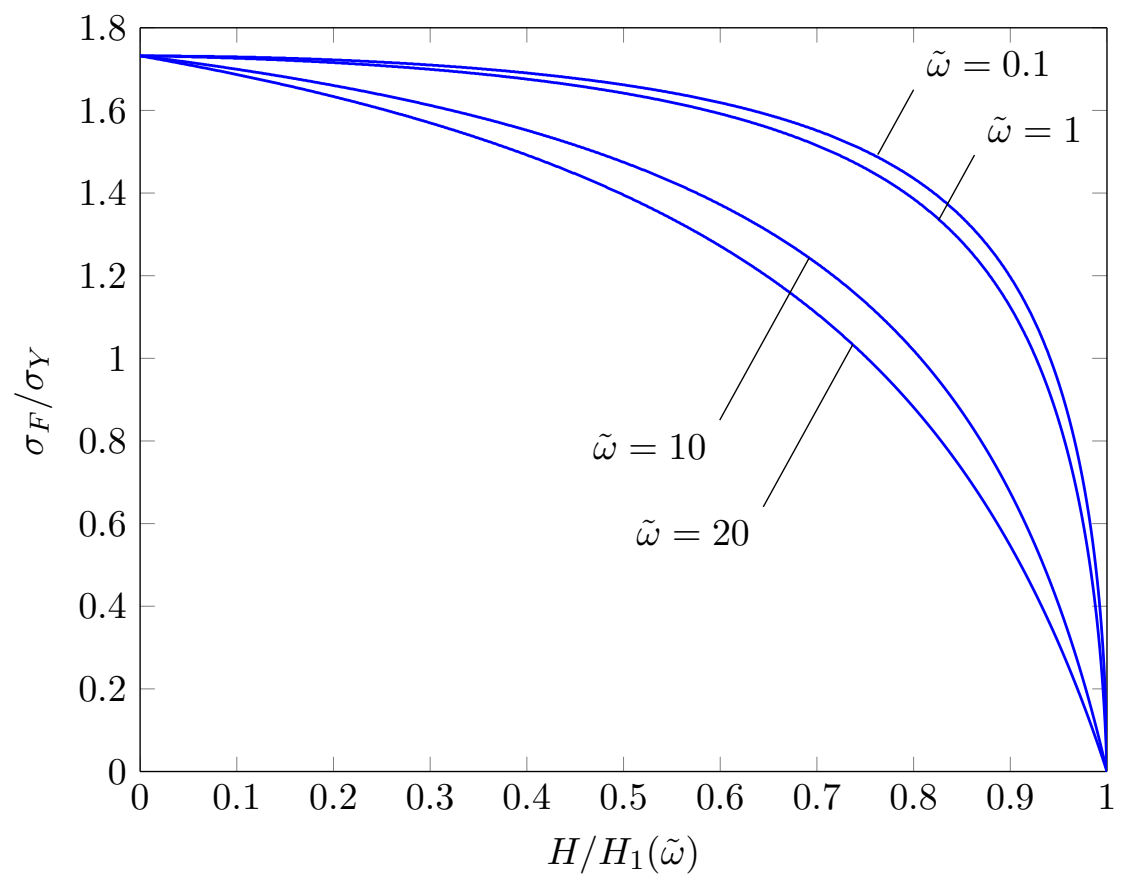

Figure 15: Shakedown map for a cylinder particle under a harmonic chemo-mechanical loading. 


\subsection{Spherical particle}

The case of a spherical particle can be studied by a similar analysis. We only report some results for a free-standing spherical particle (with radius $R$ ) submitted to a $T$-periodic flux $J(t)$ of lithium on its boundary. The shakedown domain in the $(\tilde{\omega}, H)$ is shown in Fig. 16 for a harmonic charging (red dotted line) and a smoothed square-wave charging (red solid line). The charging parameter $H$ is the maximum average concentration of injected lithium. The expression of the shakedown limit $H_{S D}(\tilde{\omega})$ in the harmonic case is

$$
H_{S D}(\tilde{\omega})=\frac{3 \sqrt{3} \sigma_{Y}}{G|\tilde{a}|}\left((\Re \zeta-3)^{2}+(\Im \zeta)^{2}\right)^{-\frac{1}{2}}
$$

where $\zeta=\hat{R} j_{0}(\hat{R}) / j_{1}(\hat{R})$ and $j_{i}$ is the spherical Bessel function of the first kind. In the limit $\tilde{\omega} \rightarrow 0$, the shakedown limit $H_{S D}(\tilde{\omega})$ in 6.48 becomes infinite, as for the stress-free cylinder particle. In free standing conditions, the spherical geometry allows for higher shakedown limits than the cylindrical geometry, see Fig. 16. As mentioned in Sect. 6.1.2, the maximum concentration of lithium in common electrode materials is in the range $10^{4}-410^{4} \mathrm{~mol} / \mathrm{m}^{3}$, which corresponds to a charging parameter $H$ in the range $5 H_{0}-20 H_{0}$. The results in Fig. 16 can be used to assess the minimum time for charging a free particle to its maximum capacity while ensuring that it remains in the elastic shakedown regime. For a smoothed square wave charging, that minimum time falls in the range $4-16 \mathrm{~h}$ for a spherical particle and in the range $10-40 \mathrm{~h}$ for a cylinder particle.

\section{Concluding remarks}

Setting aside the technical assumptions that there exists a $T$-periodic solution to 3.8 and that the dimension of $\mathcal{H}$ is finite, the main results of Sect. 4 can be informally summarized a follows: For cyclically loaded media coupling plasticity with diffusion, there is a convergence to a cyclic steady state in which the stress, the chemical potential, the flux of guest atoms and the plastic strain rate evolve in a time-periodic fashion. Some features of the cyclic steady state are path independent, i.e. independent of the initial state. This is notably the case of the stress rate, the plastic strain rate and the flux of guest atoms.

The cyclic steady-state carries a lot of relevant information for the design of structures. The most intuitive way of estimating the cyclic steady state is to resort to step-by-step incremental analysis over a sufficiently large number of cycles. More computationally efficient methods could possibly be developed by building on various techniques that have been used in standard plasticity (Maitournam et al., 2002; Spiliopoulos and Panagiotou, 2012, Peigney and Stolz, 2001, 2003). Such 'direct' methods would tentatively reduce the computational effort needed to establish Bree-type diagrams recording the main features of the cyclic steady state as a function of the loading parameters.

As mentioned in Sect. 4.3 , the particular case of elastic shakedown is of special interest both from mechanical and chemical considerations. The two-step method suggested 


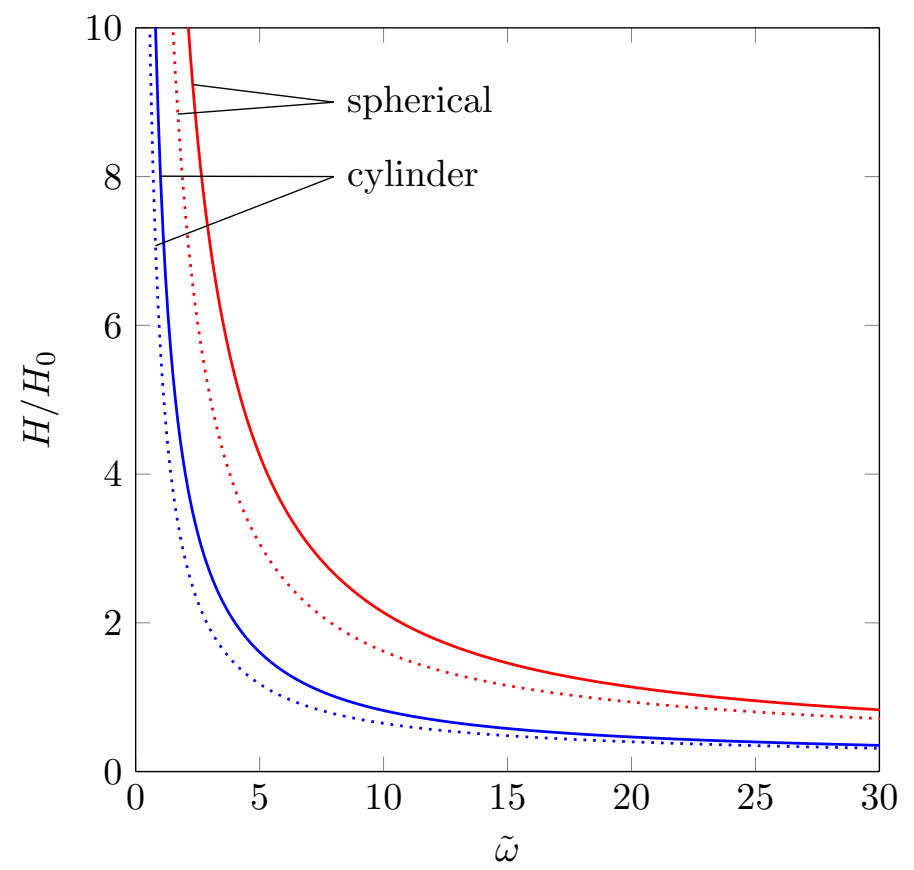

Figure 16: Shakedown maps for a free spherical particle (red) and a free cylinder particle (blue). Case of a harmonic charging (dotted line) and a smoothed square-wave charging (solid line).

in Sect. 5 allows one to estimate the set of loading parameters for which shakedown occurs - without resorting to step-by-step calculation of the chemo-mechanical evolution in elasto-plasticity. Interestingly, that two-step method is relatively simple to use: The first step is completely disconnected from plasticity and consists in finding the cyclic steadystate for the fictitious elasto-diffusive problem. Once the elasto-diffusive problem is solved, the second step is performed without any reference to diffusion. For the simple geometry considered in Sect. 6, most of the needed calculations could be performed in closed-form. For more complex geometries, adequate numerical techniques would be necessary: the fictitious elasto-diffusive problem could possibly be solved by combining a Fourier decomposition with a finite-element method (for solving the harmonic problem). Step 2 could be performed numerically by observing that it amounts to solve a convex programming problem, for which efficient algorithms (such as interior-point methods) are available.

The results presented in this paper could tentatively contribute to the design of Li-ion batteries with improved efficiency and cycle life. In that regard, it would be interesting to investigate whether similar shakedown conditions could be obtained in the finite strain setting: This would be relevant for the study of emerging silicon-based electrode materials as the lithium-induced volumetric change in those materials can reach values up to $300 \%$. Coupling with temperature is also an important topic to be explored. Furthermore, it would 
be interesting to study whether the results presented could be extended to more complex cases such as non quadratic free energies, concentration-dependent diffusion coefficients, or non-associative plasticity.

\section{Appendix A. Proof of property 1}

Let $\boldsymbol{\Sigma}=(\boldsymbol{\sigma}, \mu)$ and $\boldsymbol{\Sigma}^{\prime}=\left(\boldsymbol{\sigma}^{\prime}, \mu^{\prime}\right)$ be two solutions of 3.8$)$. We have

$$
\frac{1}{2} \frac{d\left\|\boldsymbol{\Sigma}(t)-\boldsymbol{\Sigma}^{\prime}(t)\right\|^{2}}{d t}=\left\langle\boldsymbol{\Sigma}(t)-\boldsymbol{\Sigma}^{\prime}(t), \dot{\boldsymbol{\Sigma}}(t)-\dot{\boldsymbol{\Sigma}}^{\prime}(t)\right\rangle .
$$

Using (3.8) with $(\boldsymbol{\rho}, \nu)=\left(\boldsymbol{\sigma}(t)-\boldsymbol{\sigma}^{\prime}(t), \mu(t)-\mu^{\prime}(t)\right) \in \mathcal{K}_{0}$ gives

$$
\begin{aligned}
\left\langle\dot{\boldsymbol{\Sigma}}(t), \boldsymbol{\Sigma}(t)-\boldsymbol{\Sigma}^{\prime}(t)\right\rangle= & -\int_{\Omega} \dot{\boldsymbol{\varepsilon}}^{p}:\left(\boldsymbol{\sigma}-\boldsymbol{\sigma}^{\prime}\right) d \omega-\int_{\Omega} \partial \psi(\nabla \mu) \cdot\left(\nabla \mu-\nabla \mu^{\prime}\right) d \omega \\
& +\int_{\Gamma_{u}} \dot{\boldsymbol{U}} \cdot\left(\boldsymbol{\sigma}-\boldsymbol{\sigma}^{\prime}\right) \cdot \boldsymbol{n} d S-\int_{\Gamma_{j}} J\left(\mu-\mu^{\prime}\right) d S
\end{aligned}
$$

where $\dot{\boldsymbol{\varepsilon}}^{p} \in \partial \phi(\boldsymbol{\sigma})$. Since $\boldsymbol{\Sigma}^{\prime}(t)$ is also a solution of $(3.8)$, we have similarly

$$
\begin{aligned}
\left\langle\dot{\boldsymbol{\Sigma}}^{\prime}(t), \boldsymbol{\Sigma}(t)-\boldsymbol{\Sigma}^{\prime}(t)\right\rangle= & -\int_{\Omega} \dot{\boldsymbol{\varepsilon}}^{p^{\prime}}:\left(\boldsymbol{\sigma}-\boldsymbol{\sigma}^{\prime}\right) d \omega-\int_{\Omega} \partial \psi\left(\nabla \mu^{\prime}\right) \cdot\left(\nabla \mu-\nabla \mu^{\prime}\right) d \omega \\
& +\int_{\Gamma_{u}} \dot{\boldsymbol{U}} \cdot\left(\boldsymbol{\sigma}-\boldsymbol{\sigma}^{\prime}\right) \cdot \boldsymbol{n} d S-\int_{\Gamma_{j}} J\left(\mu-\mu^{\prime}\right) d S
\end{aligned}
$$

where $\dot{\boldsymbol{\varepsilon}}^{p \prime} \in \partial \phi\left(\boldsymbol{\sigma}^{\prime}\right)$. Subtracting A.3 from A.2 and using A.1 gives

$$
\frac{1}{2} \frac{d\left\|\boldsymbol{\Sigma}(t)-\boldsymbol{\Sigma}^{\prime}(t)\right\|^{2}}{d t}=-\mathcal{D}_{p}-\mathcal{D}_{d}
$$

where

$$
\mathcal{D}_{p}=\int_{\Omega}\left(\dot{\varepsilon}^{p}-\dot{\varepsilon}^{p \prime}\right):\left(\boldsymbol{\sigma}-\boldsymbol{\sigma}^{\prime}\right) d \omega
$$

and

$$
\mathcal{D}_{d}=\int_{\Omega}\left(\partial \psi(\nabla \mu)-\partial \psi\left(\nabla \mu^{\prime}\right)\right) \cdot\left(\nabla \mu-\nabla \mu^{\prime}\right) d \omega .
$$

Since $\psi$ is convex, $\partial \psi$ is monotone i.e. $\left(\partial \psi(\nabla \mu)-\partial \psi\left(\nabla \mu^{\prime}\right)\right) \cdot\left(\nabla \mu-\nabla \mu^{\prime}\right) \geq 0$ (Van Tiel, 1984). Hence

$$
\mathcal{D}_{d} \geq 0 \text {. }
$$

In a similar fashion, the convexity of $\phi$ yields

$$
\mathcal{D}_{p} \geq 0 \text {. }
$$

It follows that $\frac{d\left\|\boldsymbol{\Sigma}(t)-\boldsymbol{\Sigma}^{\prime}(t)\right\|^{2}}{d t} \leq 0$ which leads to property 1 . 


\section{Appendix B. Proof of property 2}

We now proceed to prove property 2, using the same notations as in Appendix A If $\left\|\boldsymbol{\Sigma}(t)-\boldsymbol{\Sigma}^{\prime}(t)\right\|$ is constant for $t \geq t_{1}$, then Eqs. (A.4), A.6) and (A.7) imply that $\mathcal{D}_{p}=\mathcal{D}_{d}=0$ for all $t \geq t_{1}$. Consequently

$$
\left(\dot{\boldsymbol{\varepsilon}}^{p}(\boldsymbol{x}, t)-\dot{\boldsymbol{\varepsilon}}^{p \prime}(\boldsymbol{x}, t)\right):\left(\boldsymbol{\sigma}(\boldsymbol{x}, t)-\boldsymbol{\sigma}^{\prime}(\boldsymbol{x}, t)\right)=0
$$

and

$$
\left(\partial \psi(\nabla \mu(\boldsymbol{x}, t))-\partial \psi\left(\nabla \mu^{\prime}(\boldsymbol{x}, t)\right) \cdot\left(\nabla \mu(\boldsymbol{x}, t)-\nabla \mu^{\prime}(\boldsymbol{x}, t)\right)=0\right.
$$

at each location $\boldsymbol{x}$ and each time $t \geq t_{1}$. Since $\psi$ is strictly convex, Eq. (B.2) implies that $\nabla \mu(\boldsymbol{x}, t)=\nabla \mu^{\prime}(\boldsymbol{x}, t)$, i.e.

$$
\mu(\boldsymbol{x}, t)-\mu^{\prime}(\boldsymbol{x}, t)=f(t)
$$

for some function $f$.

Using (3.8) with the field $(\boldsymbol{\rho}, \nu)=\left(\dot{\boldsymbol{\sigma}}(t)-\dot{\boldsymbol{\sigma}}^{\prime}(t), \dot{\mu}(t)-\dot{\mu}^{\prime}(t)\right) \in \mathcal{K}_{0}$ and taking (B.3) into account, we obtain

$$
\left\langle\dot{\boldsymbol{\Sigma}}(t), \dot{\boldsymbol{\Sigma}}(t)-\dot{\boldsymbol{\Sigma}}^{\prime}(t)\right\rangle=-\int_{\Omega} \dot{\boldsymbol{\varepsilon}}^{p}:\left(\dot{\boldsymbol{\sigma}}-\dot{\boldsymbol{\sigma}}^{\prime}\right) d \omega+\int_{\Gamma_{u}} \dot{\boldsymbol{U}} \cdot\left(\dot{\boldsymbol{\sigma}}-\dot{\boldsymbol{\sigma}}^{\prime}\right) \cdot \boldsymbol{n} d S-\int_{\Gamma_{J}} J \dot{f} d S .
$$

We have similarly

$$
\left\langle\dot{\boldsymbol{\Sigma}}^{\prime}(t), \dot{\boldsymbol{\Sigma}}(t)-\dot{\boldsymbol{\Sigma}}^{\prime}(t)\right\rangle=-\int_{\Omega} \dot{\boldsymbol{\varepsilon}}^{p^{\prime}}:\left(\dot{\boldsymbol{\sigma}}-\dot{\boldsymbol{\sigma}}^{\prime}\right) d \omega+\int_{\Gamma_{u}} \dot{\boldsymbol{U}} \cdot\left(\dot{\boldsymbol{\sigma}}-\dot{\boldsymbol{\sigma}}^{\prime}\right) \cdot \boldsymbol{n} d S-\int_{\Gamma_{J}} J \dot{f} d S
$$

Subtracting (B.5) from (B.4) gives

$$
\left\|\dot{\boldsymbol{\Sigma}}(t)-\dot{\boldsymbol{\Sigma}}^{\prime}(t)\right\|^{2}=-\int_{\Omega}\left(\dot{\boldsymbol{\sigma}}-\dot{\boldsymbol{\sigma}}^{\prime}\right):\left(\dot{\boldsymbol{\varepsilon}}^{p}-\dot{\boldsymbol{\varepsilon}}^{p \prime}\right) d \omega
$$

At this point it is necessary to distinguish between viscoplasticity and rate-independent plasticity. In the case of viscoplasticity, Eq. (B.1) together with the strict convexity of the dissipation potential (outside of the elasticity domain) implies that $\dot{\boldsymbol{\varepsilon}}^{p}=\dot{\boldsymbol{\varepsilon}}^{p^{\prime}}$. It follows from B.6 that $\left\|\dot{\boldsymbol{\Sigma}}(t)-\dot{\boldsymbol{\Sigma}}^{\prime}(t)\right\|=0$. Hence $\dot{\boldsymbol{\sigma}}-\dot{\boldsymbol{\sigma}}^{\prime}=0$ and $\dot{\mu}-\dot{\mu}^{\prime}=0$, i.e. $\boldsymbol{\sigma}-\boldsymbol{\sigma}^{\prime}$ and $\mu-\mu^{\prime}$ are time-independent fields. We can conclude that the function $f$ in $($ B.3 is actually a constant. Since $\boldsymbol{\Sigma}$ and $\boldsymbol{\Sigma}^{\prime}$ are both in $\mathcal{K}(\boldsymbol{f}, \boldsymbol{T}, M)$, we necessarily have $\boldsymbol{\Sigma}-\boldsymbol{\Sigma}^{\prime} \in \mathcal{K}_{0}$.

The case of rate-independent plasticity is slightly more involved and can be handled using arguments introduced by Halphen (1978): From the principle of maximum dissipation 2.10, we have $\left(\boldsymbol{\sigma}-\boldsymbol{\sigma}^{\prime}\right): \dot{\boldsymbol{\varepsilon}}^{p} \geq 0$ and $\left(\boldsymbol{\sigma}^{\prime}-\boldsymbol{\sigma}\right): \dot{\boldsymbol{\varepsilon}}^{p \prime} \geq 0$. Hence the relation (B.1) implies that

$$
0=\left(\boldsymbol{\sigma}-\boldsymbol{\sigma}^{\prime}\right): \dot{\boldsymbol{\varepsilon}}^{p}=\left(\boldsymbol{\sigma}-\boldsymbol{\sigma}^{\prime}\right): \dot{\boldsymbol{\varepsilon}}^{\prime \prime} .
$$

For any $d t$ we have

$$
\left(\boldsymbol{\sigma}^{\prime}(t+d t)-\boldsymbol{\sigma}^{\prime}(t)\right): \dot{\boldsymbol{\varepsilon}}^{p}=\left(\boldsymbol{\sigma}^{\prime}(t+d t)-\boldsymbol{\sigma}(t)\right): \dot{\boldsymbol{\varepsilon}}^{p}+\left(\boldsymbol{\sigma}(t)-\boldsymbol{\sigma}^{\prime}(t)\right): \dot{\boldsymbol{\varepsilon}}^{p} .
$$


In the right-hand side of $(\mathrm{B} .8)$, the first term is negative because of the principle of maximum dissipation (2.10). The second term vanishes because of (B.7). Taking the limit $d t \rightarrow 0$ in $B .8$, we thus obtain that

$$
\dot{\boldsymbol{\sigma}}^{\prime}: \dot{\boldsymbol{\varepsilon}}^{p} \leq 0
$$

A similar reasoning can be used to show that

$$
\dot{\sigma}: \dot{\boldsymbol{\varepsilon}}^{\prime \prime} \leq 0
$$

Moreover, the normality flow rule 2.11 implies that

$$
\dot{\boldsymbol{\sigma}}: \dot{\boldsymbol{\varepsilon}}^{p}=\dot{\boldsymbol{\sigma}}^{\prime}: \dot{\boldsymbol{\varepsilon}}^{p \prime}=0 .
$$

Combining the relations B.9 - B.11) shows that $\left(\dot{\boldsymbol{\sigma}}-\dot{\boldsymbol{\sigma}}^{\prime}\right):\left(\dot{\boldsymbol{\varepsilon}}^{p}-\dot{\boldsymbol{\varepsilon}}^{p \prime}\right) \geq 0$. Using (B.6) we thus have

$$
0 \leq\left\|\dot{\boldsymbol{\Sigma}}(t)-\dot{\boldsymbol{\Sigma}}^{\prime}(t)\right\|^{2}=-\int_{\Omega}\left(\dot{\boldsymbol{\sigma}}-\dot{\boldsymbol{\sigma}}^{\prime}\right):\left(\dot{\boldsymbol{\varepsilon}}^{p}-\dot{\boldsymbol{\varepsilon}}^{p^{\prime}}\right) \leq 0
$$

It follows that $\left\|\dot{\boldsymbol{\Sigma}}(t)-\dot{\boldsymbol{\Sigma}}^{\prime}(t)\right\|=0$. From this point, one can conclude as in the case of viscoplasticity.

\section{Appendix C. Proof of Opial's lemma}

For the sake of self-containment, we give in this section a proof of Opial's lemma. We recall the assumptions of that lemma:

(i) $\left\{\left\|u_{n}-f\right\|\right\}$ converges for all $f \in F$,

(ii) the limit of every (weakly) convergent subsequence of $\left\{u_{n}\right\}$ is in $F$

We wish to show that the whole sequence $\left\{u_{n}\right\}$ weakly converges. We first prove that the limit of any weakly converging subsequence is uniquely defined. To that purpose, consider two converging subsequences (denoted by $\left\{u_{\varphi(n)}\right\}$ and $\left.\left\{u_{\varphi^{\prime}(n)}\right\}\right)$ and let $\left(f, f^{\prime}\right)$ be their respective weak limits, i.e.

$$
\lim _{n \rightarrow \infty}\left\langle u_{\varphi(n)}-f, g\right\rangle=0, \quad \lim _{n \rightarrow \infty}\left\langle u_{\varphi^{\prime}(n)}-f^{\prime}, g\right\rangle=0
$$

for any $g \in \mathcal{H}$. We set

$$
R(f)=\lim _{n \rightarrow \infty}\left\|u_{\varphi(n)}-f\right\|, R\left(f^{\prime}\right)=\lim _{n \rightarrow \infty}\left\|u_{\varphi^{\prime}(n)}-f^{\prime}\right\| .
$$

The limits in (C.2) exist because of the assumption $(i)$. For each $n$ we have

$$
\left\|u_{\varphi(n)}-f\right\|^{2}=\left\|u_{\varphi(n)}-f^{\prime}+f^{\prime}-f\right\|^{2}=\left\|u_{\varphi(n)}-f^{\prime}\right\|^{2}+\left\|f^{\prime}-f\right\|^{2}+2\left\langle u_{\varphi(n)}-f^{\prime}, f^{\prime}-f\right\rangle .
$$


Taking the limit $n \rightarrow \infty$ and using (C.1) as well as (C.2), we obtain

$$
R(f)^{2}=R\left(f^{\prime}\right)^{2}+\left\|f^{\prime}-f\right\|^{2} .
$$

Using a similar reasoning with $\left\{u_{\varphi^{\prime}(n)}\right\}$ gives

$$
R\left(f^{\prime}\right)^{2}=R(f)^{2}+\left\|f^{\prime}-f\right\|^{2} .
$$

Combining (C.3) and (C.4) gives $f=f^{\prime}$. The weak limit of any weakly converging subsequence is thus unique. Since the unit ball is compact for the weak topology (Rudin, 1991) and the sequence $\left\{u_{n}\right\}$ is bounded (by $(i)$ ), it follows that the whole sequence $\left\{u_{n}\right\}$ weakly converges to $f$. By $(i i)$, we have $f \in F$.

\section{References}

Ahn, Y. J., Bertocchi, E., Barber, J., 2008. Shakedown of coupled two-dimensional discrete frictional systems. J. Mech. Phys. Solids 56, 3433-3440.

Baillon, J., Haraux, A., 1977. Comportement à l'infini pour les équations d'évolution avec forcing périodique. Arch. Rational Mech. Anal. 67, 101-109.

Bower, A. F., Guduru, P. R., Sethuraman, V. A., 2011. A finite strain model of stress, diffusion, plastic flow, and electrochemical reactions in a lithium-ion half-cell. J. Mech. Phys. Solids 59, 804-828.

Brassart, L., Zhao, K., Suo, Z., 2013. Cyclic plasticity and shakedown in high-capacity electrodes of lithium-ion batteries. Int. J. Solids. Struct. 50, 1120-1129.

Bree, J., 1967. Elastic-plastic behaviour of thin tubes subjected to internal pressure and intermittent high-heat fluxes with application to fast-nuclear-reactor fuel elements. J. Strain Anal. Eng. Des. 2, 226-238.

Cheng, Y.-T., Verbrugge, M. W., 2009. Evolution of stress within a spherical insertion electrode particle under potentiostatic and galvanostatic operation. J. Power Sources 190, 453-460.

Christensen, J., Newman, J., 2006. Stress generation and fracture in lithium insertion materials. J. Solid State Electr. 10, 293-319.

Constantinescu, A., Dang Van, K., Maitournam, M., 2003. A unified approach for high and low cycle fatigue based on shakedown concepts. Fatigue Fract. Eng. Mater. Struct. $26,561-568$.

Cui, Z., Gao, F., Qu, J., 2012. A finite deformation stress-dependent chemical potential and its applications to lithium ion batteries. J. Mech. Phys. Solids 60, 1280-1295. 
Frederick, C., Armstrong, P., 1966. Convergent internal stresses and steady cyclic states of stress. J. Strain Anal. Eng. Des. 1, 154-159.

Haftbaradaran, H., Gao, H., 2012. Ratcheting of silicon island electrodes on substrate due to cyclic intercalation. Appl. Phys. Lett. 100, 121907.

Halphen, B., 1978. Steady cycles and shakedown in elastic-viscoplastic and plastic structures. In: Structures et matériaux sous chargement cyclique. Association amicale des ingénieurs anciens éleves de l'E.N.P.C., pp. 203-229.

Hill, R., 1950. The mathematical theory of plasticity. Oxford university press.

Huggins, R. A., Nix, W. D., 2000. Decrepitation model for capacity loss during cycling of alloys in rechargeable electrochemical systems. Ionics 6, 57-63.

Johnson, C., Mercier, B., Nedelec, J. C., 1978. Convergence to a periodic solution in perfect plasticity. In: Structures et matériaux sous chargement cyclique. Association amicale des ingénieurs anciens éleves de l'E.N.P.C., pp. 253-255.

Klarbring, A., Barber, J., Spagnoli, A., Terzano, M., 2017. Shakedown of discrete systems involving plasticity and friction. Eur. J. Mech. A 64, 160-164.

Koiter, W. T., 1960. General problems for elastic solids. Progress in solid mechanics.

Larché, F., Cahn, J. W., 1973. A linear theory of thermochemical equilibrium of solids under stress. Acta Metallurgica 21, 1051-1063.

Maier, G., Pastor, J., Ponter, A., Weichert, D., 2003. Direct methods of limit and shakedown analysis. Numerical and computational methods 3, 637-684.

Maitournam, H., Pommier, B., Thomas, J., 2002. Détermination de la réponse asymptotique d'une structure anélastique sous chargement cyclique. C.R. Mecanique 330, 703708.

Melan, E., 1936. Theorie statisch unbestimmter systeme aus ideal-plastischen baustoff. Sitz.Berl.Ak.Wiss. 145, 195-218.

Nguyen, Q. S., 2003. On shakedown analysis in hardening plasticity. J. Mech. Phys. Solids $51,101-125$.

Opial, Z., 1967. Weak convergence of the sequence of successive approximations for nonexpansive mappings. Bull. Amer. Math. Soc. 73, 591-597.

Peigney, M., 2010. Shakedown theorems and asymptotic behaviour of solids in non-smooth mechanics. Eur. J. Mech. A 29, 784-793. 
Peigney, M., 2014a. On shakedown of shape memory alloys structures. Ann. Solid Struct. Mech. 6, 17-28.

Peigney, M., 2014b. Shakedown of elastic-perfectly plastic materials with temperaturedependent elastic moduli. J. Mech. Phys. Solids 71, 112-131.

Peigney, M., Stolz, C., 2001. Approche par contrôle optimal des structures élastoviscoplastiques sous chargement cyclique. C.R.Acad.Sci. Paris, II 329, 643-648.

Peigney, M., Stolz, C., 2003. An optimal control approach to the analysis of inelastic structures under cyclic loading. J. Mech. Phys. Solids 51, 575-605.

Pham, D. C., 2008. On shakedown theory for elastic-plastic materials and extensions. J. Mech. Phys. Solids 56, 1905-1915.

Pham, D. C., 2017. Consistent limited kinematic hardening plasticity theory and pathindependent shakedown theorems. Int. J. Mech. Sci. 130, 11-18.

Reimers, J. N., Dahn, J., 1992. Electrochemical and in situ x-ray diffraction studies of lithium intercalation in $\mathrm{li}_{x} \mathrm{CoO}_{2}$. J. Electrochem. Soc. 139, 2091-2097.

Rudin, W., 1991. Functional analysis. McGraw-Hill.

Spagnoli, A., Terzano, M., Barber, J., Klarbring, A., 2017. Non-linear programming in shakedown analysis with plasticity and friction. J. Mech. Phys. Solids 104, 71-83.

Spiliopoulos, K. V., Panagiotou, K. D., 2012. A direct method to predict cyclic steady states of elastoplastic structures. Comput. Method. Appl. Mech. Eng. 223, 186-198.

Swaminathan, N., Balakrishnan, S., George, K., 2016. Elasticity and size effects on the electrochemical response of a graphite, li-ion battery electrode particle. J. Electrochem. Soc. 163, A488-A498.

Symonds, P. S., 1951. Shakedown in continuous media. J. Appl. Mech. 18, 85-89.

Van Tiel, J., 1984. Convex analysis. John Wiley.

Wesfreid, E., 1981. Asymptotic behaviour of an elastoviscoplastic material submitted to a cyclic load. Proc. Roy. Soc. Edin. A 89, 189-199.

Wong, R., 2001. Asymptotic approximations of integrals. SIAM.

Yang, H., Fan, F., Liang, W., Guo, X., Zhu, T., Zhang, S., 2014. A chemo-mechanical model of lithiation in silicon. J. Mech. Phys. Solids 70, 349-361.

Zhang, X., Shyy, W., Sastry, A. M., 2007. Numerical simulation of intercalation-induced stress in li-ion battery electrode particles. J. Electrochem. Soc. 154, A910-A916. 
Zhao, K., Pharr, M., Vlassak, J. J., Suo, Z., 2010. Fracture of electrodes in lithium-ion batteries caused by fast charging. J. Appl. Phys. 108, 073517.

Zhao, K., Pharr, M., Vlassak, J. J., Suo, Z., 2011. Inelastic hosts as electrodes for highcapacity lithium-ion batteries. J. Appl. Phys. 109, 016110. 